\title{
The protective role of TLR6 in a mouse model of asthma is mediated by IL-23 and IL-17A
}

\author{
Ana Paula Moreira, ${ }^{1}$ Karen A. Cavassani, ${ }^{1}$ Ugur B. Ismailoglu, ${ }^{1}$ Rikki Hullinger, ${ }^{1}$ Michael P. Dunleavy, ${ }^{1}$ \\ Darryl A. Knight,, ${ }^{2}$ Steven L. Kunkel, ${ }^{1}$ Satoshi Uematsu, ${ }^{3}$ Shizuo Akira, ${ }^{3}$ and Cory M. Hogaboam ${ }^{1}$
}

1Department of Pathology, University of Michigan Medical School, Ann Arbor, Michigan, USA. 2James Hogg Research Centre of the Heart and Lung Institute, University of British Columbia, Vancouver, British Columbia, Canada. ${ }^{3}$ Department of Host Defense, Osaka University, Osaka, Japan.

\begin{abstract}
TLRs are a family of receptors that mediate immune system pathogen recognition. In the respiratory system, TLR activation has both beneficial and deleterious effects in asthma. For example, clinical data indicate that TLR6 activation exerts protective effects in asthma. Here, we explored the mechanism or mechanisms through which TLR6 mediates this effect using mouse models of Aspergillus fumigatus-induced and house dust mite antigen-induced (HDM antigen-induced) chronic asthma. Tlr6 $6^{-/-}$mice with fungal- or HDM antigen-induced asthma exhibited substantially increased airway hyperresponsiveness, inflammation, and remodeling compared with WT asthmatic groups. Surprisingly, whole-lung levels of IL-23 and IL-17 were markedly lower in $T \operatorname{Tr} 6^{-/-}$versus WT asthmatic mice. $\operatorname{Tl} \boldsymbol{G}^{-/-}$DCs generated less IL-23 upon activation with lipopolysaccharide, zymosan, or curdlan. Impaired IL-23 generation in $\mathrm{Tlr}^{-/-}$mice also corresponded with lower levels of expression of the pathogen-recognition receptor dectin- 1 and expansion of Th17 cells both in vivo and in vitro. Exogenous IL-23 treatment of asthmatic $\mathrm{Tlr}^{-/-}$mice restored IL-17A production and substantially reduced airway hyperresponsiveness, inflammation, and lung fungal burden compared with that in untreated asthmatic $\mathrm{Tlr}^{-/-}$mice. Together, our data demonstrate that TLR6 activation is critical for IL-23 production and Th17 responses, which both regulate the allergic inflammatory response in chronic fungal-induced asthma. Thus, therapeutics targeting TLR6 activity might prove efficacious in the treatment of clinical asthma.
\end{abstract}

\section{Introduction}

Asthma is a chronic inflammatory disease that predisposes individuals to reversible episodes of airway obstruction and shortness of breath (1). In atopic patients, inhaled environmental allergens incite Th2-type cytokine and recruitment of inflammatory cells into the lungs (1). Asthmatics with hypersensitivity to Aspergillus fumigatus exhibit Th2-type cell recruitment and increased risk of fungal persistence or colonization $(2,3)$. A major initiating event in asthmatic airway disease appears to revolve around the nature of the interaction between DCs and T cells (reviewed in refs. 4, 5). DC and $\mathrm{T}$ cell interactions favor the generation of Th2 cells or those T cells that generate IL-4, IL-9, and IL-13 during allergic inflammation, thereby setting off a cascade of immune-activating events that lead to changes not only in the contractile nature of the smooth muscle cells surrounding the airway but also the overall structure of the airway itself, with profound changes in remodeling. Key $\mathrm{T}$ cell activation pathways are required for the containment of the Th2 response, and these include Th1 cells and the Th1-type cytokines they generate, $\mathrm{T}$ regulatory cells via the generation of IL-10 and TGF- $\beta$, and Th17 cells, which generate IL-17 and IL-22 (6). Whether this last $\mathrm{T}$ cell type has beneficial or deleterious roles in allergic airway disease is presently not completely resolved (7).

While not all asthmatic responses are driven by allergens, they are among the most potent stimuli in the asthmatic airway (1). Asthma-associated allergens include fungi such as A. fumigatus (2) and house dust mite (HDM) antigens. Aside from patients with allergic bronchopulmonary aspergillosis, who exhibit fungal colonization and asthmatic disease, sensitization to A. fumigatus affects up to $25 \%$ of all asthmatics and is correlated with incidence

Conflict of interest: The authors have declared that no conflict of interest exists. Citation for this article: J Clin Invest. 2011;121(11):4420-4432. doi:10.1172/JCI44999. and severity of disease $(2,3,8)$. Recently, David Denning and colleagues reported that antifungal therapy attenuates certain disease features in patients with severe asthma with fungal sensitization (9). The manner in which A. fumigatus spores or conidia interact with allergic inflammatory cells involves a number of cell-associated recognition receptors, of which the most intensely studied are the TLRs. Nevertheless, to date it is not readily apparent whether TLR activation during asthmatic responses to fungus such as A. fumigatus inhibits or amplifies the allergic inflammatory response in asthma (10).

Three extracellular TLRs that recognize A. fumigatus have been identified: TLR2, TLR4, and TLR6. Among these TLRs, only TLR6 is upregulated following allergenic sensitization to A. fumigatus in mice (11). TLR6 forms a heterodimer with TLR2 for the recognition of diacyl lipopeptides, zymosan, and lipoteichoic acid (12). TLR2 also forms a heterodimer with TLR1, a complex that recognizes triacyl lipopeptides (12). Signaling through both complexes leads to NF- $\kappa \mathrm{B}$ activation and cytokine generation (13). The putative role of TLR6 during clinical asthma is still unknown, but clinical studies have indicated that single nucleotide polymorphisms in TLR6 have a role in the protection from asthma (14-16). More recently, protective effects of TLR6 polymorphisms in asthma were associated with increased expression and greater mononuclear cell generation of Th1-type cytokines (17). In contrast, one TLR6 polymorphism that appears to lead to defective TLR6 expression and/or function is associated with increased incidence of asthma (17). Finally, a polymorphism in TLR6 has also been associated with susceptibility to invasive aspergillosis after allogeneic stem cell transplantation (18).

Our objective in the present study was to determine the role of TLR6 in the development and maintenance of experimental asthma. The study demonstrated that $\operatorname{Tlr}^{-/-}$mice developed an exag- 

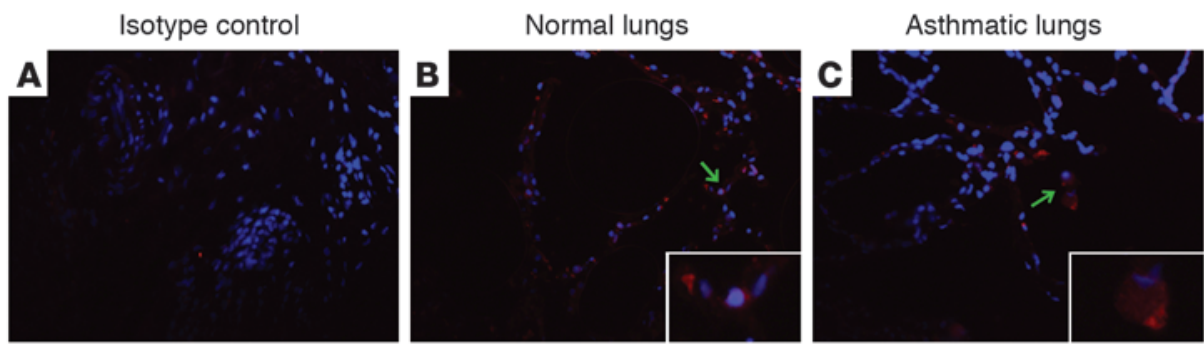

Figure 1

TLR6 expression in asthmatic lungs. TLR6 expression (red) was evaluated using immunofluorescence in normal lungs (B) or in the lungs of asthmatic patients with severe disease (C). Nuclei stained with DAPI (blue) in all photomicrographs and isotype control are demonstrated in A. Arrows indicate the presence of TLR6. Original magnification, $\times 400$. Photomicrographs are representative of 6 different normal or 6 different asthmatic lungs.

gerated form of fungal- and HDM antigen-induced allergic airway disease. Surprisingly, the major differences were in levels of IL-23 and IL-17A between asthmatic WT and Tlr6 $6^{-/-}$mice. Zymosan- or LPS-activated $T l r 6^{--}$DCs generated substantially less IL-23 compared with similarly activated WT DCs and failed to promote Th17 differentiation. The reduced responsiveness of $T l r 6^{--}$DCs to zymosan was due to the near absence of dectin-1 expression. The exogenous supplementation of IL-23 to asthmatic Tlr $6^{-/-}$mice promoted latency-associated peptide-TGF- $\beta$ (LAP-TGF- $\beta$ ) expression by DCs and the expansion of Th17 cells, and reduced the fungal burden, airway hyperresponsiveness, and allergic inflammatory response in these mice compared with untreated Tlr6 $6^{-/}$mice. Thus, TLR6 is required for the elaboration of IL-23 and IL-17 during experimental asthma, and its absence exacerbates asthma severity.

\section{Results}

Mononuclear and alveolar epithelial cells in whole-lung samples from asthmatic patients express TLR6. Chun and colleagues (19) recently profiled TLR protein expression on peripheral blood mononuclear cells in asthmatics and observed that, unlike TLR1, TLR2, and TLR9, TLR6 expression was significantly lower in asthmatic patients compared with controls. Moreover, this receptor has been associated with decreased susceptibility for the development of atopic asthma (16). Because these studies did not examine the type of cells expressing TLR6 in whole-lung tissue, we first investigated whether TLR6 was present in asthmatic lungs. TLR6 expression in histological lung sections from asthmatic patients with severe disease compared with normal lung sections is shown in Figure 1. All asthmatic lung sections exhibited intense perivascular and peribronchial inflammatory infiltration by polymorphonuclear and mononuclear cells. TLR6 expression was analyzed by immunofluorescence, and this receptor was largely observed in mononuclear cells in the inflammatory foci (Figure 1C). It was also noted that TLR6 was present on alveolar epithelial cells in asthmatic tissue sections and in normal lung sections (Figure 1, B and C). Thus, mononuclear immune cells and alveolar epithelial cells in asthmatic tissue expressed TLR6, leading us to address the specific role of this receptor in experimental asthma.

The absence of TLR6 increases methacholine-induced airway resistance, inflammation, and goblet cell metaplasia during experimental asthma. To investigate the role of TLR6 in experimental asthma, WT and $\mathrm{Tlr}^{-/}$mice were sensitized and challenged with A. fumigatus antigens and live conidia (or spores) or HDM antigen, as represented
WT group challenged for 6 weeks with HDM antigens exhibited methacholine-induced airway resistance, but $\operatorname{Tlr}^{-1-}$ mice challenged in a similar manner with these antigens showed a significant increase in airway resistance above baseline following methacholine administration (Figure 2D). A greater number of immune and inflammatory cells were observed in bronchoalveolar lavage (BAL) samples from HDM antigen-challenged $\operatorname{Tlr} 6^{-/-}$mice (Figure 2E), and an increased percentage of BAL polymorphonuclear cells was present in knockout mice compared with WT mice at day 7 after conidia challenge (Figure 2F). A histological examination of whole-lung tissue sections revealed that the peribronchial inflammatory response in $\operatorname{Tlr}^{-1-}$ mice was markedly more intense than that in the WT groups at days 15 and 30 after conidia challenge (Figure 2F). Although the inflammation appeared to be similar between the WT and $\operatorname{Tlr}^{-/}$groups challenged with HDM antigen, goblet cell metaplasia was more intense in the knockout group (Figure 2F). A morphometric analysis of the cellularity of small airways at day 30 after conidia challenge confirmed that significantly greater numbers of cells were present in the airways of $\operatorname{Tlr}^{--}$mice compared with the WT group (Figure 2G). The absence of Tlr6 also affected the magnitude of the goblet cell metaplasia in fungal asthma, since transcripts of mucus-associated genes, namely Muc5ac and Clca3 (Gob5), were significantly elevated in the knockout group compared with the WT group (Figure $2 \mathrm{H}$ ). Collectively, these data demonstrate that TLR6 modulates several major features of experimental fungaland HDM antigen-induced asthma, including airway hyperreactivity, inflammation, and remodeling.

TLR6 regulates pattern recognition receptor-induced IL-23 production by $D C$ from asthmatic mice. To further explore the mechanism through which TLR6 regulated the inflammatory response in experimental asthma, ELISA was used to determine cytokine levels in whole-lung samples from the WT and $\operatorname{Tlr}^{-/}$groups at various times prior to and after the conidia challenge. Whole-lung IL-10 levels were significantly lower in the $\mathrm{Tlr}^{-/}$group compared with the WT group at day 15 after conidia challenge, but immunoreactive levels of this cytokine and IL- 13 , IL-4, TGF- $\beta$, and IL-12p70 were similarly significantly increased in both WT and $\mathrm{Tlr}^{-1-}$ mice at day 30 after conidia challenge compared with the levels of these cytokines prior to the conidia challenge (Figure 3A). IFN- $\gamma$ levels were similar at all times prior to and after the conidia challenge in both groups of mice (Figure 3A). However, analysis of IL-23 levels in whole-lung samples showed that this cytokine was significantly less abundant in the knockout groups compared with the WT groups at days 15 and 

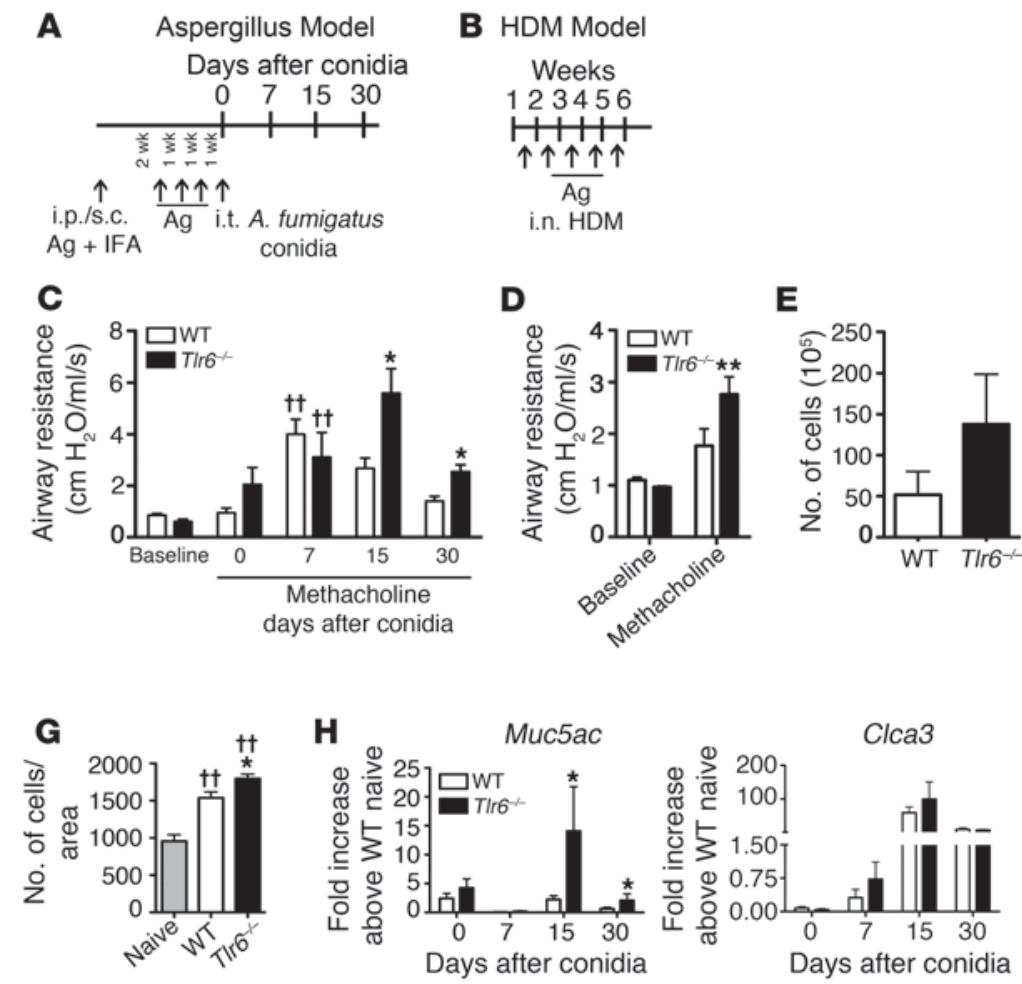

\section{B HDM Model \\ Weeks \\ 123456

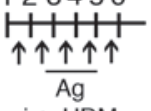 \\ i.n. HDM}

\section{D}

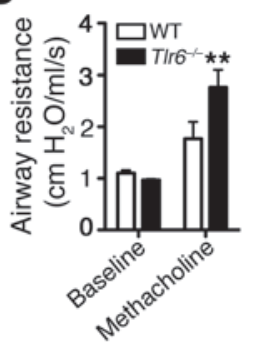

E

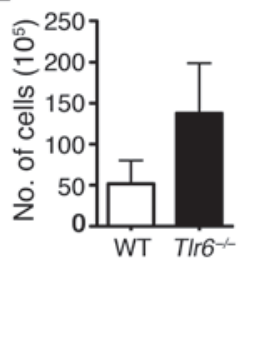

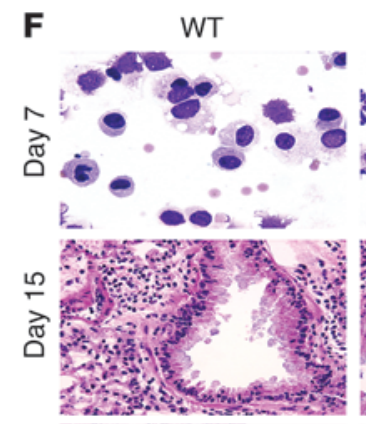

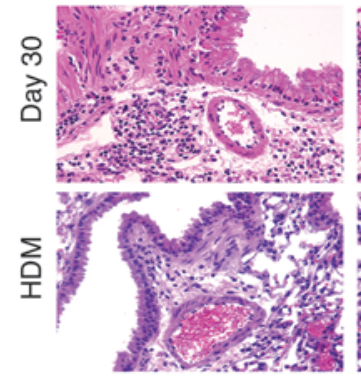

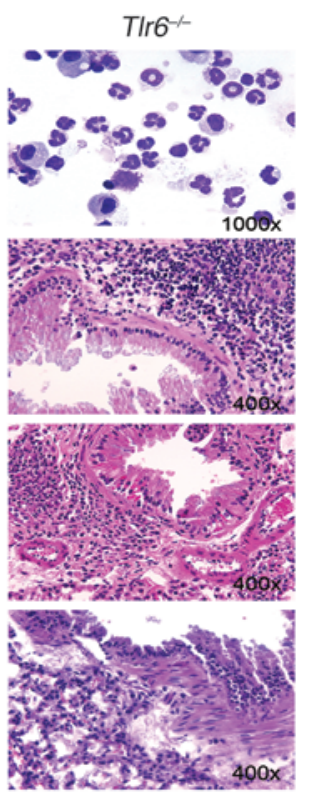

\section{Figure 2}

TIr6 absence increases airway resistance and inflammation during fungal- and HDM antigen-induced asthmatic disease. (A and B) Schematic representation of the A. fumigatus- and HDM antigen-allergic models. Ag, antigen. (C) Airway resistance at days $0,7,15$, and 30 after conidia challenge and (D) 72 hours after HDM antigen challenge in WT or TIr6 ${ }^{-1-}$ allergic mice. (E) Number of bronchoalveolar cells in HDM antigen-allergic mice. (F) Bronchoalveolar cells and lung sections of asthmatic mice stained with H\&E. Original magnification, $\times 400$ (day 15, day 30, HDM); $\times 1000$ (day 7). (G) Morphometric analysis of changes in percentage of cells present around the airways of naive WT, A. fumigatus-sensitized WT, and TIr6 ${ }^{-1-}$ mice at day 30 after conidia challenge. (H) Transcript expression for Muc5ac and Clca3 in whole lung. Data represent mean \pm SEM of 2 independent experiments, and $n=4-5$ mice/group. ${ }^{\star} P<0.05$ compared with WT mice; ${ }^{\star \star} P<0.05$ compared with baseline; ${ }^{\dagger+} P<0.05$ compared with day 0 or naive mice.

30 after conidia challenge (Figure 3A). In addition, Il23 transcripts were significantly reduced in the lungs of $\operatorname{Tr} 6^{-/-}$mice challenged with HDM antigen compared with the WT group (Supplemental Figure 1; supplemental material available online with this article; doi:10.1172/JCI44999DS1). IL-17A levels were lower in the Tlr6-/group compared with the WT group at all times prior to and after conidia challenge, and this difference reached statistical significance at day 30 after conidia challenge (Figure 3A). Together, these data suggested that there was a defect in IL-23 and IL-17A generation in $\operatorname{Tr} 6^{-/}$mice following the induction of experimental asthma.

Because DCs generate IL-23 in response to several pattern recognition receptor-specific (PRR-specific) stimuli (20), we next addressed the role of TLR6 in IL-23 generation by DCs from asthmatic mice. Myeloid DCs were generated from BM taken from both the WT and $\operatorname{Tlr}^{-/-}$asthmatic groups and activated with various PRR-specific stimuli. Transcript levels of Il23a at 6 hours after stimulus were determined by TaqMan, and this analysis showed that Il23 generation was absent in $\mathrm{Tlr}^{-/-}$DCs following their exposure to PAM2Cys (a TLR2/6 ligand), as expected. However, Tlr6 ${ }^{-/}$ DCs also expressed significantly less $I l 23$ a transcript following activation with zymosan (a dectin-1 ligand), LPS (a TLR4 ligand), or
CpG (a TLR9 ligand) compared with similarly activated WT DCs (Figure 3B). The defect in Il23a transcript expression appeared to be PRR specific, since Tlr6 $6^{-1-}$ DCs expressed greater Il23a than WT DCs following exposure to PAM3cys (a TLR2/1 ligand). Using an ELISA analysis of cell-free supernatants from DC cultures, IL-23 levels were detected at significantly lower levels in samples from Tlr6 $6^{-/-}$DCs exposed to LPS or zymosan compared with similarly treated WT DCs (Figure 3C). Although IL-12p70 levels were significantly decreased in $\mathrm{Tlr}^{-{ }^{--}}$versus WT DCs activated with zymosan, LPS induced similar levels of this cytokine in both types of DCs (Figure 3C). Because curdlan has greater specificity than zymosan for dectin-1, WT and Tlr6 $6^{--}$DCs were also exposed to this $\beta$-glucan with medium alone or a blocking antibody directed against dectin-1. IL-23 levels were markedly reduced in the supernatant of $\mathrm{Tlr}^{-/-}$DCs stimulated with medium or curdlan compared with WT cells (Figure 3D). Moreover, the presence of anti-dectin- 1 antibody significantly decreased IL-23 production in cultures of WT DCs and abolished IL-23 generation in Tlr6 $6^{-1-}$ DC cultures stimulated with curdlan (Figure 3D). Thus, these results indicated that TLR6 expression by DCs from asthmatic mice was necessary for the generation of IL-23 at the transcript and protein levels. 
A
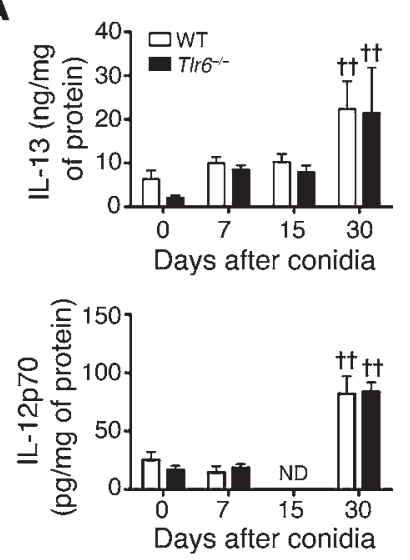

B

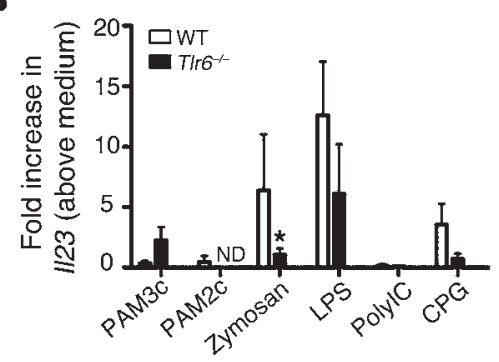

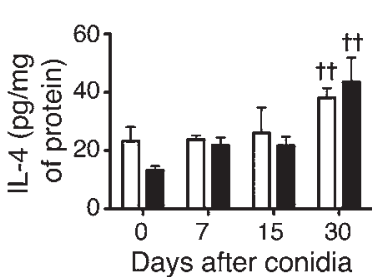

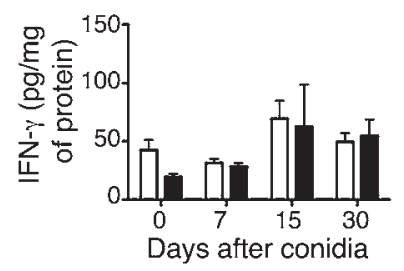

C

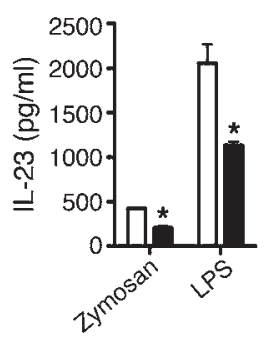

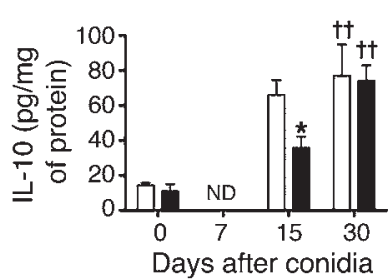
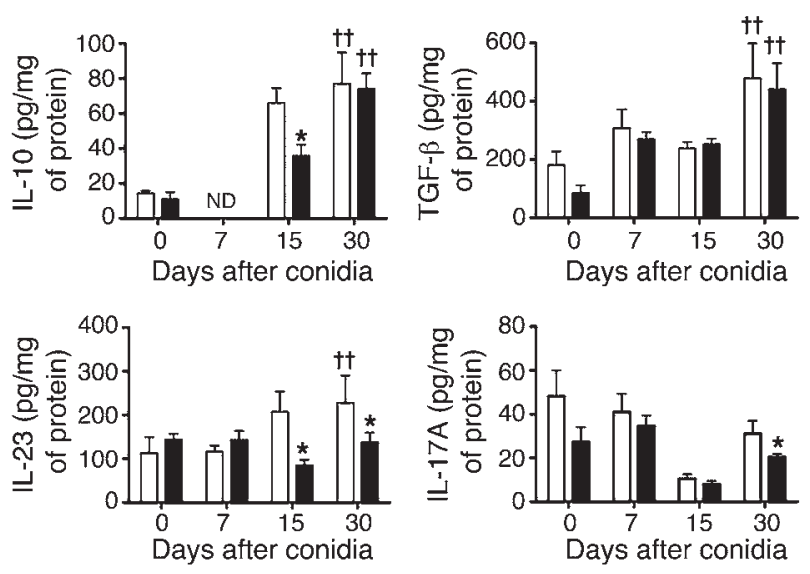

D
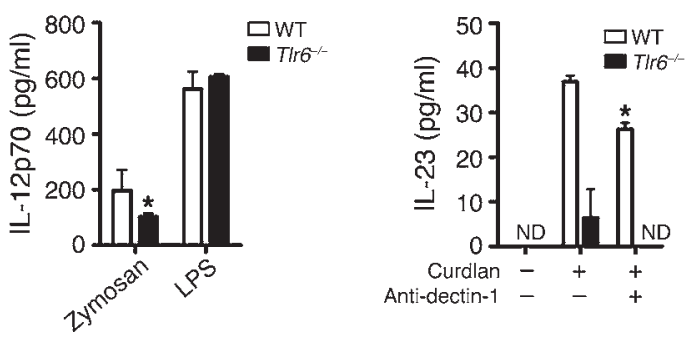

Figure 3

IL-23 levels are reduced in asthmatic TIr6 $6^{-/-}$mice. (A) Cytokine levels in whole-lung samples of asthmatic mice from days 0 to 30 after conidia challenge. (B) Transcript expression of II23 in BMDCs from asthmatic WT or TIr6-1- mice stimulated for 6 hours with dectin-1 or TLR ligands. (C) IL-23 or IL-12p70 levels in the supernatant of BMDCs stimulated for 24 hours with TLRs or dectin-1 ligands or (D) stimulated with curdlan with medium alone or anti-dectin-1. Data represent mean \pm SEM of 2 independent experiments. ${ }^{\dagger \dagger} P<0.05$ compared with day 0 ; ${ }^{*} P<0.05$ compared with WT control mice.

TLR6 is necessary for dectin-1 expression and activation in the lung and isolated DCs. While the dectin-1 agonists zymosan and curdlan were potent stimuli for IL-23 expression in WT DCs, they were poor agonists for $I l 23$ a expression in $T l r 6^{-/-}$DCs. This observation led us to further investigate the extracellular signals that promoted dectin-1 expression in DCs and define the consequence of Tlr6 deficiency on dectin-1 expression in vivo. To first explore the nature of signals that promote dectin-1 expression in DCs, BMDCs from naive WT mice were exposed to medium alone, LPS, PAM2Cys, PAM3Cys, or zymosan for 24 hours prior to TaqMan analysis of Clec7a expression. PAM2Cys, PAM3Cys, and zymosan all significantly induced Clec $7 a$ expression by approximately 6- to 20 -fold above Clec7a levels in DCs exposed to medium alone (Figure 4A). Interestingly, recombinant IL-23 (rIL-23) induced Clec7a expression in DCs by approximately 30-fold above levels of this C-type lectin in DCs treated with medium alone (Figure 4A). Transcript levels for $\mathrm{Clec} 7 \mathrm{a}$ were determined by TaqMan in whole lungs from both groups of mice prior to and after conidia challenge, and the results are summarized in Figure 4B. At all time points in this model, Clec7a transcript expression was lower in the knockout group compared with the WT group, and this difference reached statistical significance at day 15 after conidia challenge (Figure 4B). Immunohistochemistry was used to confirm the deficit in dectin-1 expression in $\operatorname{Tlr}^{-/-}$mice at day 15 after conidia challenge, and this C-type lectin PRR was markedly reduced in the lung sections from $\mathrm{Tlr}^{-1-}$ mice compared with similar lung sections from WT mice
(Figure 4C). To further explore the mechanisms by which TLR6 activation leads to dectin-1 expression, we investigated whether Tlr $6^{-/-}$DCs exhibited altered expression of PU.1, c-Jun, and SP1 (i.e., important transcription factors for the induction of dectin-1). rIL-23 increased transcript expression of Clec $7 a$ (dectin-1) and Sfpi1 (PU.1) in allergic Tlr6-1- BMDCs when compared with cells stimulated with medium alone, LPS, zymosan, or rIL-12 (Figure 4D). Further, the in vivo treatment of $\mathrm{Tr}^{-/-}$mice with rIL-23 promoted a significant increase in both Clec $7 a$ and Spfi1 compared with nontreated allergic mice (Figure 4E). Together, these data demonstrated that TLR6 ligands and IL-23 are potent inducers of Spfi1 and Clec7a expression by DCs. The deficient production of IL-23 by $\operatorname{Tlr}^{-/-}$cells might contribute to the decreased levels of dectin-1 in the lungs of allergic mice.

To further investigate the role of TLR6 and dectin-1 in IL-23 generation by immune cells during asthma, mixed splenocyte cultures from WT and $T l r 6^{-/-}$asthmatic mice were exposed to medium alone or A. fumigatus antigen in the presence or absence of anti-dectin-1 antibody. Constitutive IL-23 generation in mixed splenocyte cultures from $\mathrm{Tlr}^{-/-}$mice was below the limits of detection, and IL-23 levels were significantly decreased in cultures of $\operatorname{Tlr}^{-/-}$splenocytes exposed to A. fumigatus antigens when compared with the appropriate WT group (Figure 4F). Dectin-1 antibody blockade markedly reduced or abolished IL-23 levels in WT and $\operatorname{Tlr}^{-/-}$splenocyte cultures, respectively. Thus, IL-23 generation in mixed splenocyte cultures from asthmatic mice was TLR6- and dectin-1-dependent 

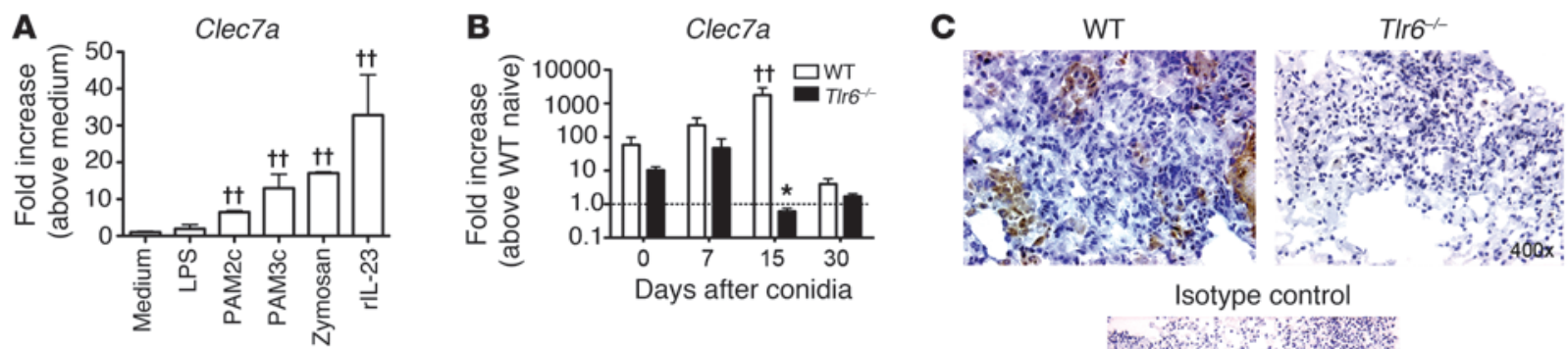

Days after conidia

Isotype control
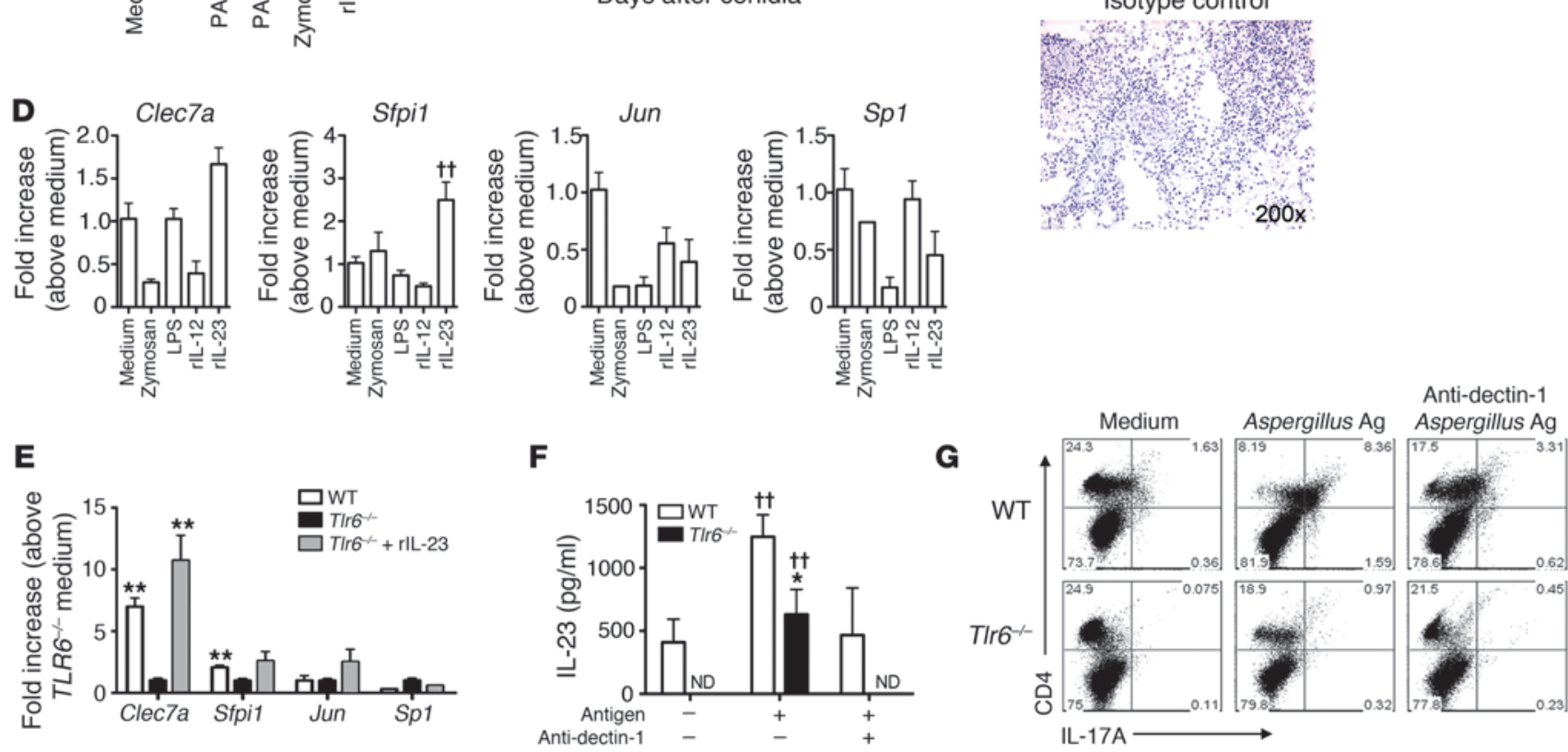

\section{Figure 4}

TLR6 regulates dectin-1 expression in asthmatic lungs. (A) Transcript expression for Clec7a (dectin-1) in naive BMDCs stimulated for 6 hours with dectin-1, TLR ligand, or rlL-23 or (B) in whole lungs of allergic mice. (C) Dectin-1 expression (stained brown) was evaluated in whole-lung tissue sections from WT and TIr6-/- mice at day 15 after conidia challenge using immunohistochemistry. Original magnification, $\times 200$ (isotype control); $\times 400$ (WT and TIr6-/-). (D) Transcript expression for Clec7a, Sfpi1, Jun, and Sp1 in allergic Tlr6 ${ }^{-/-}$BMDCs stimulated for 6 hours with medium, zymosan, LPS, rIL-12, or rIL-23 or (E) allergic BMDCs from WT and TIr6 ${ }^{-1-}$ mice treated in vivo with rIL-23. (F) IL-23 levels and (G) percentage of CD3 T cells expressing CD4 and intracellular IL-17A were analyzed in spleen cultured with medium alone, $A$. fumigatus antigen, or anti-dectin-1 and A. fumigatus antigen from WT and TIr6-/- asthmatic mice at day 15 after conidia challenge. Data represent mean \pm SEM of 2 independent experiments, and $n=4-5$ mice/group. ${ }^{\star} P<0.05$ compared with WT mice; ${ }^{\star \star} P<0.05$ compared with TIr6 ${ }^{-/-}$mice; ${ }^{t \dagger} P<0.05$ compared with day 0 or medium.

with and without $A$. fumigatus antigen challenge. Since IL-23 has been shown to be involved in the differentiation and expansion of Th17 cells (20), our next avenue of investigation was to determine whether TLR6 deficiency affected the expansion of these cells during fungal asthma. Thus, the percentage of $\mathrm{CD}^{+} \mathrm{CD}^{+}$ cell-expressing intracellular IL-17A was analyzed by flow cytometry in this coculture. A. fumigatus antigen increased the percentage of IL-17A-expressing cells among WT cells (Figure 4G), but the antibody-mediated blockade of dectin-1 decreased the percentage of IL-17A-expressing T cells by 2.5 -fold when compared with cells exposed to antigen alone. We also observed that few IL-17-positive cells were present in the Tlr6-deficient culture stimulated by A. fumigatus antigen. Dectin-1 blockage resulted in an approximately 7-fold decrease in intracellular IL-17 expression in $\mathrm{Tlr}^{-1}$ cultures. Thus, Tlr6 expression was necessary for Th17 cell expansion during experimental asthma.

Tlr6 $6^{-1-}$ DCs have an impaired ability to induce Th17 cell expansion. To verify whether TLR6 affected the phenotype of T cells in vivo, flow cytometric analyses of intracellular levels of IFN- $\gamma$, IL-4, IL-17, and foxp 3 were performed in $\mathrm{CD}^{+} \mathrm{CD}^{+} \mathrm{T}$ cells in both WT and $\mathrm{Tlr} 6^{-/-}$ mice at day 30 after conidia challenge. The results are summarized in Figure 5A. We observed lower numbers of T cells expressing IFN- $\gamma$, IL-4, and foxp3 in lung-draining LNs from $\mathrm{Tlr}^{-1-}$ mice than in the WT group, but these differences did not reach statistical significance (Figure 5A). However, IL-17A-positive T cell levels were significantly lower in $\mathrm{LN}$ samples from $\mathrm{Tlr}^{-/}$mice compared with WT mice (Figure 5A). In agreement with these findings, Tlr6 $6^{-1-}$ DCs pulsed with OVA poorly induced IL-17A-producing $T$ cells when cocultured with memory Th17 differentiated in vivo from OTII transgenic mice (Supplemental Figure 2). To further evaluate the manner in which the absence of TLR6 affected Th17 differentiation in asthmatic mice, $\mathrm{CD}^{+} \mathrm{T}$ cells and $\mathrm{CD} 11 \mathrm{c}^{+} \mathrm{DCs}$ were isolated from asthmatic WT or $\mathrm{Tlr}^{-/-}$mice and cocultured with medium alone or $A$. fumigatus antigen $(2 \mu \mathrm{g} / \mathrm{ml})$. At 72 hours after the start of coculture, approximately $12 \%$ of the $\mathrm{CD}^{+} \mathrm{T}$ cells expressed IL-17A; however, this number was significantly reduced, by $50 \%$, with the addition of anti-IL-23 monoclonal antibody to $6 \%$ of total T cells (Figure $5 \mathrm{~B}$ ). The coculture of $\operatorname{Tlr}^{-/-}$DCs with 
A

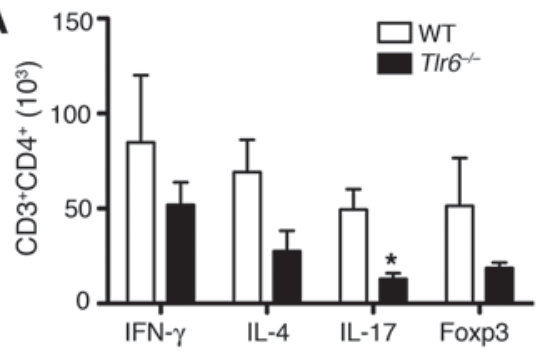

B

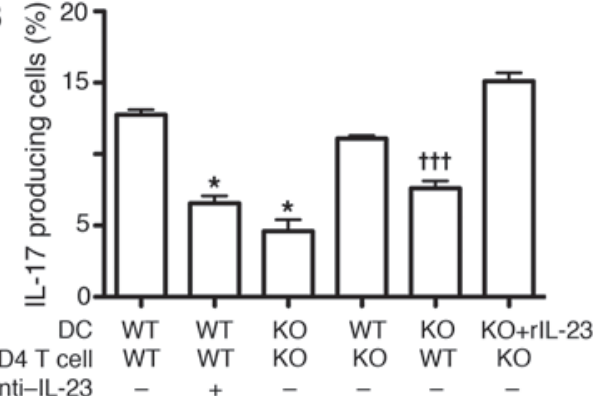

CD4 T cell WT WT KO KO WT KO
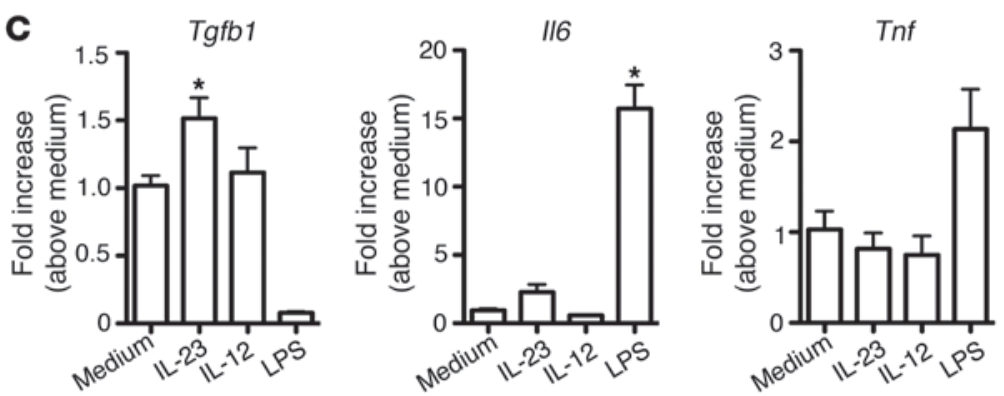

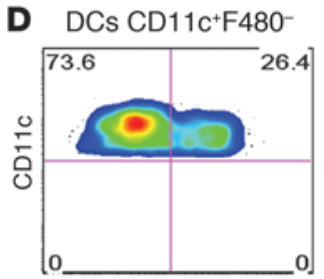

IL-23 receptor

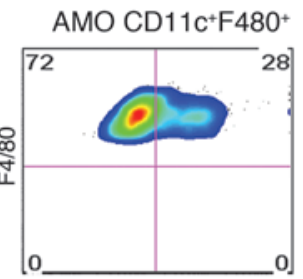

o]

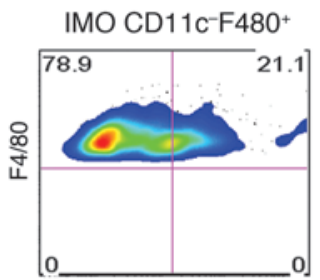

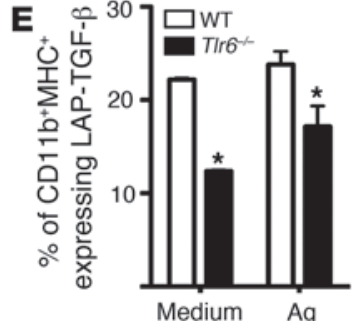

Figure 5

TIr6 ${ }^{-/}$DCs have an impaired ability to regulate Th17 cell expansion due to impaired IL-23 levels. (A) Number of $\mathrm{CD}^{+}{ }^{+} \mathrm{CD} 4^{+}$cells expressing intracellular IFN- $\gamma$, IL-4, IL-17, or foxp3 in LN from WT and TIr6-/- mice at day 30 after conidia challenge. (B) Percentage of IL-17A-producing cells in coculture of CD11 $\mathrm{C}^{+}$and $\mathrm{CD}^{+}{ }^{+}$cells isolated from spleen of WT, TIr6 $6^{-/}$, or TIr6 $6^{-/-}$mice treated with rlL-23. ${ }^{+t+} P<0.05$ compared with the cocultures of WT DCs and TIr6 ${ }^{-1-}$ CD4 T cells. (C) Transcript expression of $/ / 6, T g f b 1$, and Tnf in BMDCs from WT naive mice stimulated in vitro with medium alone or rIL-23, rIL-12, or LPS. (D) Percentage of DCs and AMO or IMO expressing IL-23 receptor in naive lungs. (E) Percentage of bead-isolated CD11c cells expressing CD11b, $\mathrm{MHCII}$, and LAP-TGF- $\beta$ in lungs from WT and $\mathrm{TIr}^{-/-}$mice at day 21 after conidia challenge cultured either with medium alone or $A$. fumigatus antigen $(\mathrm{Ag})$. Data represent 2 independent experiments and show mean $\pm \mathrm{SEM}$ of $n=5$ mice/group. ${ }^{*} P<0.05$ compared with WT under the same conditions or medium.

Tlr6 $6^{-/-}$T cells resulted in a similar percentage of IL-17A-producing $\mathrm{T}$ cells, approximately $6 \%$ of the total $\mathrm{T}$ cell population (Figure $5 \mathrm{~B}$ ). Coculturing Tlr6-deficient T cells with WT DCs did not impair the expansion of IL-17A-positive T cells, but TLR6-deficient DCs failed to efficiently expand WT IL-17A-positive T cells (Figure 5B). In cocultures of rIL-23-treated $\operatorname{Tlr}^{-/-}$DCs with untreated $\operatorname{Tlr} 6^{-/-}$ $\mathrm{T}$ cells, the percentage of IL-17A-producing T cells was restored to levels observed in WT cultures, indicating that IL-23 was a critical missing factor in cocultures containing $\operatorname{Tlr}^{-1-}$ DCs. One explanation for the important effect of rIL-23 on Th17 differentiation and expansion is highlighted in Figure 5C. In comparison with DCs treated with medium alone, the addition of rIL-23 to cultured DCs induced the expression of Tgfbl and Il6 (i.e., 2 known DCassociated activators of Th17 cells; ref. 21), but not Tnf transcripts (Figure 5C). Under these culture conditions, exogenous IL-12 did not promote the induction of any of these transcripts (compared text of $A$. fumigatus allergen, other allergens, such as OVA, HDM antigen, and cockroach allergen, were tested for their ability to promote LAP-TGF- $\beta$ expression in DCs. Regardless of the allergen examined, the percentage of LAP-TGF- $\beta$-expressing cells was significantly higher in the WT groups than in the $\mathrm{Tlr}^{-1-}$ groups (Supplemental Figure 3A). Moreover, the blockade of dectin-1 in WT BMDCs reduced the Tgfb1 transcript expression in these cells except in the presence of cockroach allergen (Supplemental Figure 3B). Finally, reduced $T g f b 1$ transcript levels were also observed when naive $\operatorname{Tlr}^{-/-}$DCs were stimulated with OVA or HDM antigen, but not cockroach antigens, compared with naive WT DCs (Supplemental Figure 3C). Thus, the absence of TLR6 expression by myeloid DCs curtailed the ability of these cells to drive Th17 differentiation and expansion to a variety of allergic antigens, most notably due to the absence of IL-23, TGF- $\beta$, and IL-6 generation, as shown in Figure 5. 
A

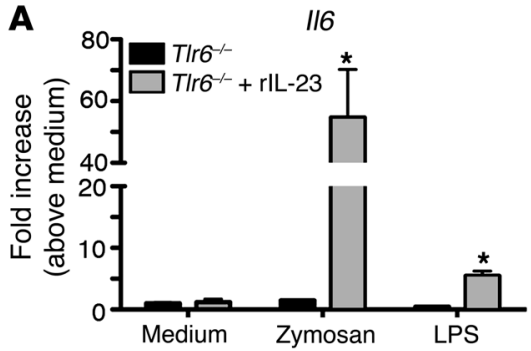

C Control

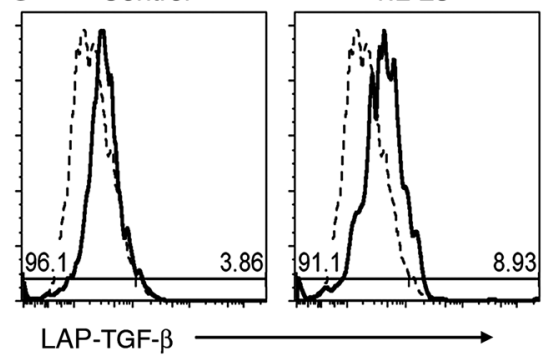

B

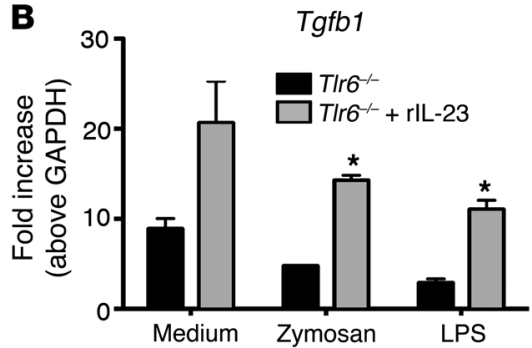

D

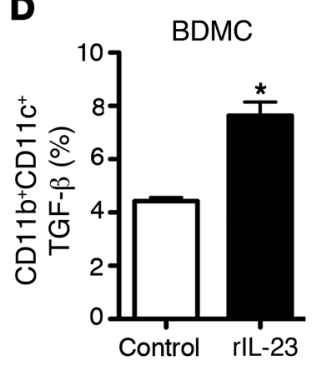

E

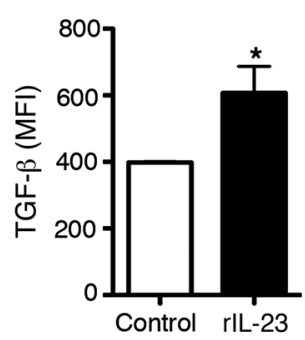

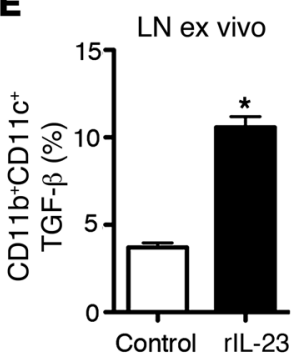

Figure 6

rlL-23 increases TGF- $\beta$ expression by DCs. Transcript expression for I/6 (A) and Tgfb1 (B) by BMDCs from allergic T/r6 ${ }^{-/-}$mice treated in vitro for 24 hours with rlL-23. (C) Histogram representing the percentage of TGF- $\beta$ cells in CD11b+CD11c+ BMDCs from asthmatic WT mice treated with rIL-23. Percentage and MFI of LAP-TGF- $\beta$ in CD11 $b^{+}$CD11 $c^{+}$cells differentiated from BM (D) or isolated from LN (E) of WT mice treated with rIL-23 from days 15 to 30 after conidia challenge. Data are mean \pm SEM of $n=5$ mice/group. ${ }^{\star} P<0.05$ compared with control group or Tlr6 ${ }^{-/-}$mice.

Exogenous i.n. IL-23 treatment of asthmatic mice significantly enhances TGF- $\beta$ expression by isolated Tlr $6^{-1}$ and WT DCs. To explore the role of IL-23 in DC activation that facilitates Th17 expansion in this model, untreated and rIL-23-treated BMDCs from $\mathrm{Tlr}^{-/-}$allergic mice were examined for their expression of $I l 6$ and $T g f b 1$. As shown in Figure 6A, cultured DCs from rIL-23-treated $\operatorname{Tr} 6^{-1}$ mice generated significantly greater quantities of Il6 transcript following in vitro zymosan or LPS activation. In addition, cultured DCs from rIL-23-treated $\mathrm{Tlr}^{-/-}$mice expressed significantly greater transcript levels of $T g f b 1$ following exposure to medium alone, zymosan, or LPS compared with DCs that did not receive rIL-23 (Figure 6B). Thus, in vivo treatment with rIL-23 significantly enhanced $\mathrm{Tlr}^{-/-}$DC expression of Il6 and Tgfb1, thereby potentially allowing these cells to promote Th17 differentiation and expansion in mice with fungal asthma.

Because rIL-23 had such a profound effect on the magnitude of the asthmatic response in $\mathrm{Tlr}^{-/-}$mice, we also examined the effect of this recombinant cytokine on the asthmatic phenotype in WT mice. rIL-23 treatment had a minor suppressive effect on methacholine-induced airway resistance, but there was little effect of this cytokine on the various parameters of airway remodeling in WT mice. However, as shown in Figure $6 \mathrm{C}$, the in vivo treatment with exogenous rIL-23 markedly increased the percentage of WT DCs expressing TGF- $\beta$. The percentage of TGF- $\beta$-positive DCs and the MFI of TGF- $\beta$ staining on these cells were significantly increased with the rIL-23 in vivo treatment (Figure 6D). Finally, the percentage of DCs expressing TGF- $\beta$ was significantly increased in lungdraining LNs from rIL-23-treated WT mice (Figure 6E). Thus, the in vivo treatment with rIL-23 significantly enhanced WT DC expression of TGF- $\beta$, but this exogenous treatment had a minor overall effect on the severity of fungal asthma in WT mice.
Exogenous i.n. IL-23 treatment decreases the severity of experimental asthma in $\mathrm{Tlr}^{-/-}$mice. To further evaluate the in vivo significance of impaired IL-23 generation due to TLR6 deficiency, asthmatic $\mathrm{Tlr}^{-/-}$mice were treated with PBS or PBS with rIL-23 via i.n. instillation from days 15 to 30 after conidia challenge. At day 30, several parameters were analyzed in the fungal asthma model, including methacholine-induced airway resistance, remodeling, inflammation, and the presence of Th17 cells, dectin-1 expression, and fungal burden. Exogenous rIL-23 significantly decreased methacholine-induced airway resistance in $T \operatorname{lr} 6^{-1-}$ mice compared with the untreated $\mathrm{Tlr}^{-/-}$group of mice (Figure $7 \mathrm{~A}$ ). Histological analysis of whole-lung sections indicated that exogenous rIL-23 treatment reduced peribronchial inflammation and collagen accumulation compared with that in the untreated $\operatorname{Tlr}^{-/-}$group (Figure $7 \mathrm{~B}$ ). Morphometric analysis of the percentage of the airway composed of collagen revealed that the airways of untreated $\operatorname{Tlr}^{-1-}$ mice expressed significantly more collagen compared with those of IL-23-treated $\mathrm{Tlr}^{-/-}$mice (Figure 7C). Supplementation i.n. of this cytokine in $\operatorname{Tlr}^{-/-}$mice also significantly increased $I l 17 a$ and Il22 transcripts in whole-lung samples (Figure 7D), the number of IL-17A-producing cells in the lung-draining LNs (Figure 7E), and IL-17A production in whole-lung samples (Figure 7F). Whole-lung samples from IL-23-treated $\mathrm{Tlr}^{-/-}$mice expressed 3-fold higher amounts of Clec 7 a transcript (Figure 7G) and 8-fold less fungal material (Figure $7 \mathrm{H}$ ) compared with those of untreated knockout mice. Thus, these data indicate that the absence of IL-23 in Tlr6 $6^{--}$ mice promotes an exaggerated form of fungal asthma characterized by increased airway resistance, inflammation, remodeling, and fungal persistence.

IL-17A and IL-22 modulate airway remodeling and inflammation during experimental asthma. Because the absence of TLR6 markedly 
A

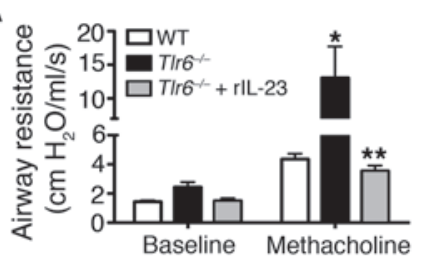

C

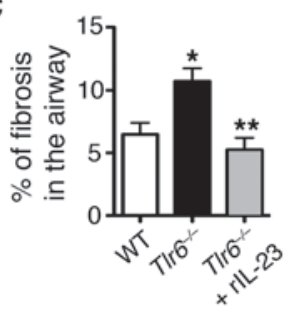

D

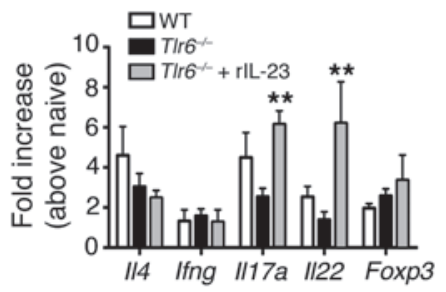

B

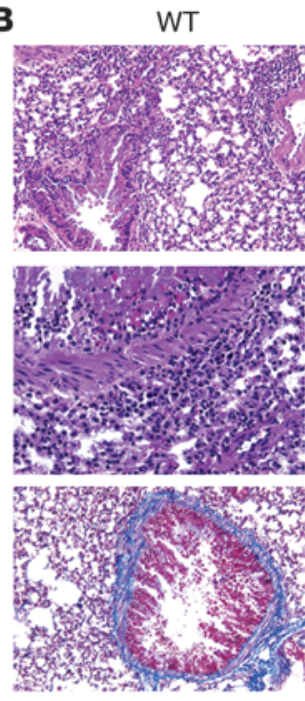

TIr6 ${ }^{-1-}$
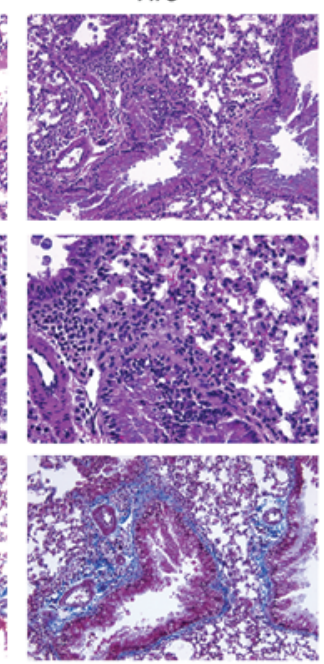

Tlr6 $6^{-\alpha}+$ rlL-23
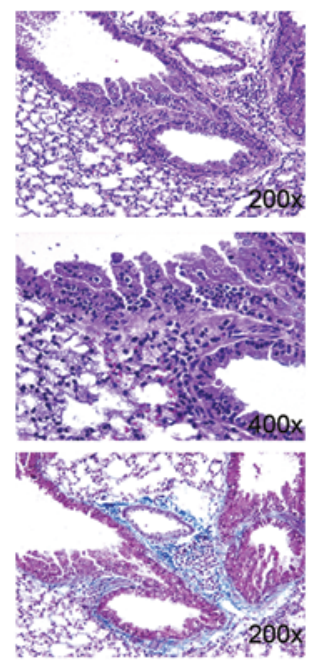

E

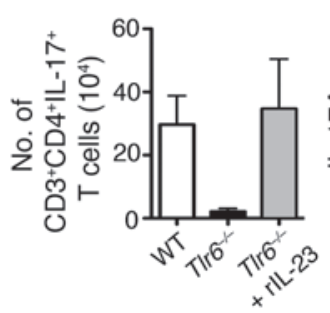

F

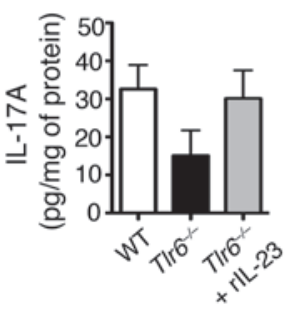

G

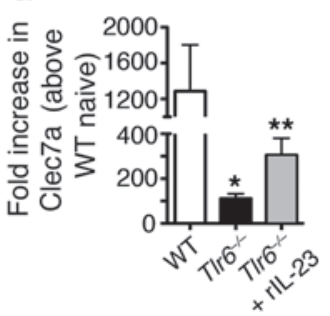

H

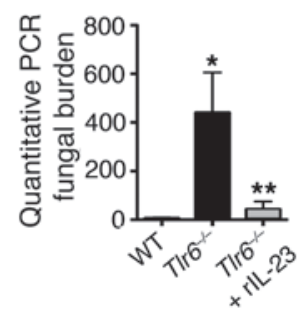

Figure 7

Exogenous IL-23 decreases asthmatic disease in TIr6 ${ }^{-/-}$mice. (A) Airway resistance at day 30 after conidia challenge in WT, TIr6 ${ }^{-/-}$, or TIr6 $6^{-/-}$mice treated with rlL-23 from days 15 to 30 after conidia challenge. (B) Lung sections of asthmatic mice stained with H\&E or Masson trichrome. Original magnification, $\times 200$ (first and third rows); $\times 400$ (second row). (C) Morphometric analysis of the percentage of the airway that contained fibrotic material. (D) Transcript expression in whole-lung samples from WT, TIr6 $6^{-1}$, or rlL-23-treated TIr6 $6^{-/-}$mice. (E) Number of CD3 $3^{+}$CD4 $4^{+}$cells expressing intracellular IL-17A in LN from asthmatic mice at day 30 after conidia challenge. (F) IL-17A levels measured by ELISA in whole lungs. (G) Transcript expression of $\mathrm{Clec7a}$ in whole lungs. (H) A. fumigatus conidia content in lung sections. Data represent 2 independent experiments and are mean \pm SEM of $n=3-5$ mice/group. ${ }^{*} P<0.05$ compared with WT mice; ${ }^{*} P<0.05$ compared with knockout mice.

impaired the expansion and maintenance of Th17 cells during fungal asthma, we next examined the involvement of IL-17A and also of IL-22, both cytokines made by Th17 cells, in this model. A. fumigatus-sensitized WT mice received anti-IL-17A monoclonal antibody, anti-IL-22 monoclonal antibody, or the appropriate isotype control antibody via i.n. instillation from days 15 to 30 after conidia challenge. At day 30, the blockade of either IL-17A or IL-22 had a minor effect on methacholine-induced airway resistance when compared with the appropriate IgG control group (data not shown). In H\&E-stained whole-lung tissue sections, the anti-IL-17A treatment partially reduced the intensity of the airway inflammatory response, but this treatment appeared to markedly enhance PAS staining of goblet cells, indicating a metaplasia of these cells (Figure 8A). Also, in histological sections, it was apparent that the blockade of IL-22 significantly increased lung inflammation when compared with that in the IgG control (Figure 8A). Quantitative analysis of mRNA by TaqMan of whole-lung samples is shown in Figure 8C, and these data confirmed that targeting IL-17A resulted in an approximate 10-fold increase in gob5 and muc5 transcripts and a 3-fold increase in coll-1 when compared with the IgG control-treated group. Conversely, these transcripts were all lower in anti-IL-22 anti- body-treated mice compared with those in the IgG control group (Figure 8B). ELISA analysis of whole-lung samples showed that anti-IL-17A antibody treatment did not change levels of IL-13, IL-25, TSLP, IL-33, or pentraxin-3 (PTX3) at day 30 after conidia challenge (Figure 8C). However, anti-IL-22 antibody treatment increased whole-lung levels of both IL-33 (a pro-Th2 mediator) and PTX3 (an inflammatory long pentraxin) in allergic mice at this time after conidia challenge (Figure $8 \mathrm{C}$ ). Thus, these results demonstrate that IL-17A and IL-22 have distinct modulatory roles in fungal asthma: IL-17A appears to regulate goblet cell metaplasia in the remodeling airways, while IL-22 has an antiinflammatory role during fungus-induced asthma.

\section{Discussion}

Previous clinical studies have addressed the presence of TLR6 polymorphisms in clinical asthma $(14,15,17)$ and invasive aspergillosis following stem cell transplant (18), and all of these studies suggested that TLR6 activity provides an important protective role in the lung. In the present study, whole-lung samples from patients with severe asthma exhibited strong TLR6 expression, particularly in mononuclear and alveolar epithelial cells. The significance of TLR6 expression in the lung was explored after the induction of 
A
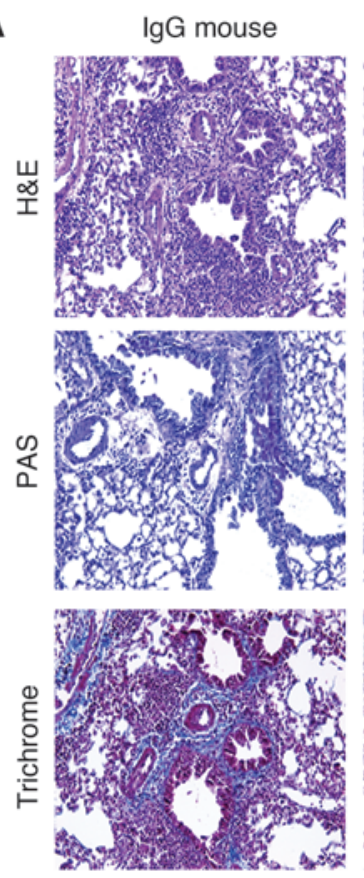

B

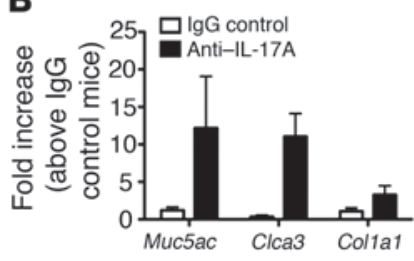

Anti-IL-17A
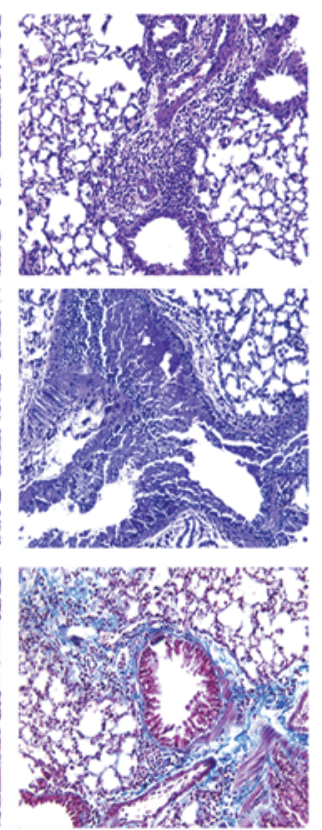
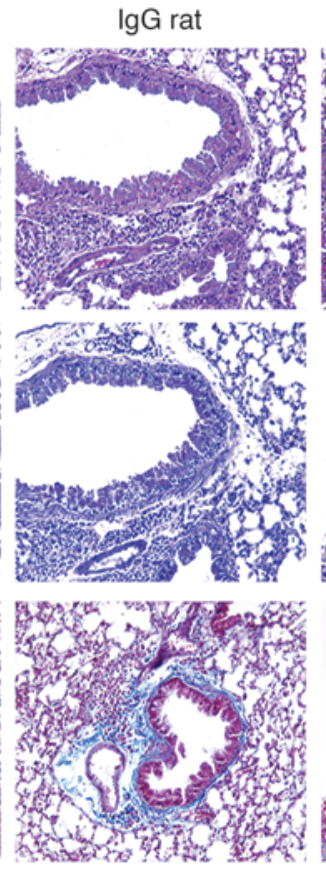

Anti-IL-22
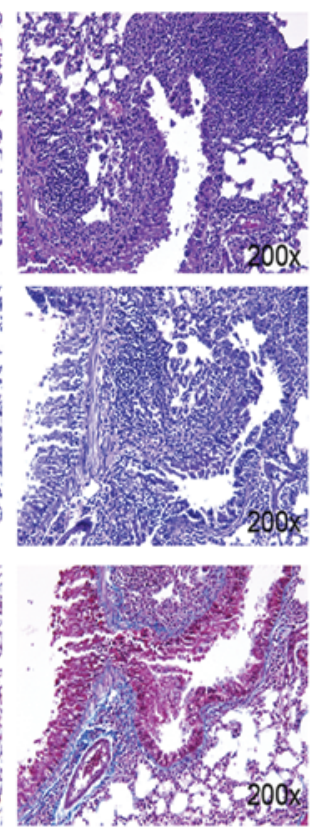
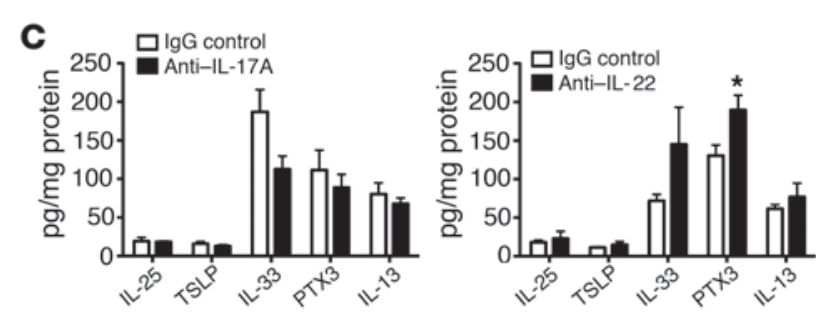

\section{Figure 8}

IL-17 and IL-22 protect mice from airway remodeling during fungal-induced asthmatic disease. (A) Lung sections at day 30 after conidia challenge in WT A. fumigatus-allergic mice treated with IgG control, anti-IL-17A, or anti-IL-22 blocking antibodies. Original magnification, $\times 200$. (B) Transcript expression for Muc5ac, Clca3, and Col1a1 in whole lungs. (C) Cytokine levels in whole-lung samples of asthmatic mice. Data represent mean \pm SEM of 5 mice/group. ${ }^{*} P<0.05$ compared with WT mice.

fungal- and HDM antigen-induced asthma in $\mathrm{Tlr}^{-/-}$mice, and these mice exhibited exaggerated features of disease, including airway resistance, inflammation, and remodeling, compared with WT mice, particularly during the chronic phase of this model (i.e., $\geq 15$ days after conidia challenge). In the absence of TLR6, DCs failed to generate IL-23 upon activation with TLR4-, dectin-1-, and TLR9-specific ligands. In fact, DCs and other lung-associated cells exhibited a marked impairment in dectin-1 expression. Tlr6 $6^{-1-}$ DCs failed to promote Th17 expansion and proliferation both in vitro and in vivo. Moreover, the exogenous treatment of $\operatorname{Tlr}^{-1-}$ mice with rIL-23 significantly increased the number of Th17 cells and the production of IL-17A and IL-22 in the lungs, and resulted in a significant decrease in the lung fungal burden compared with that in untreated $\mathrm{Tlr}^{-/-}$mice. Since this exogenous rIL-23 treatment also decreased airway resistance and inflammation in $\operatorname{Tlr}^{-/-}$mice, this study indicates that TLR6 provides a protective effect via the regulation of IL- 6 and TGF- $\beta$ expression by DCs and promotion of Th17 during asthmatic responses to A. fumigatus.

$\operatorname{Tl} 6$ was originally cloned and characterized by Akira and colleagues (22) and found to be highly homologous with TLR1. TLR6, like TLR1, forms a functional heterodimer with TLR2, thereby markedly expanding the repertoire of ligands that are recognized by TLR2. Of the 10 known human TLR members, TLR 6 is 1 of 2 (the other is TLR10) TLRs found to have strong associations with asthma susceptibility. Independent studies in both adult (14, 15) and pediatric (17) patient cohorts have confirmed that variations in TLR6 have relevance to the pathogenesis of asthma. The most recent studies by Kormann and colleagues (17) revealed that protective polymorphisms in TLR6 promoted Th1-type cytokine expression. In the present study, both immune and nonimmune cells expressed TLR6 in biopsy material from severe asthmatics, and it was clear that alveolar epithelial cells were positive for TLR6 in asthmatic and normal tissue sections. The significance of TLR6 expression by human alveolar epithelial cells is not presently clear, but activation of these cells via TLR6-specific ligands might favor immunoregulatory effects mainly in chronic asthma, like those observed in DCs in our study.

Previous studies have demonstrated that the administration of Mycoplasma fermentans-related synthetic MALP-2 (a TLR2/6 agonist) into established models of allergic asthma ameliorated many of the features of disease (23-25) via the induction of a Th1-type cytokine response. Although whole-lung IFN- $\gamma$ levels varied with 


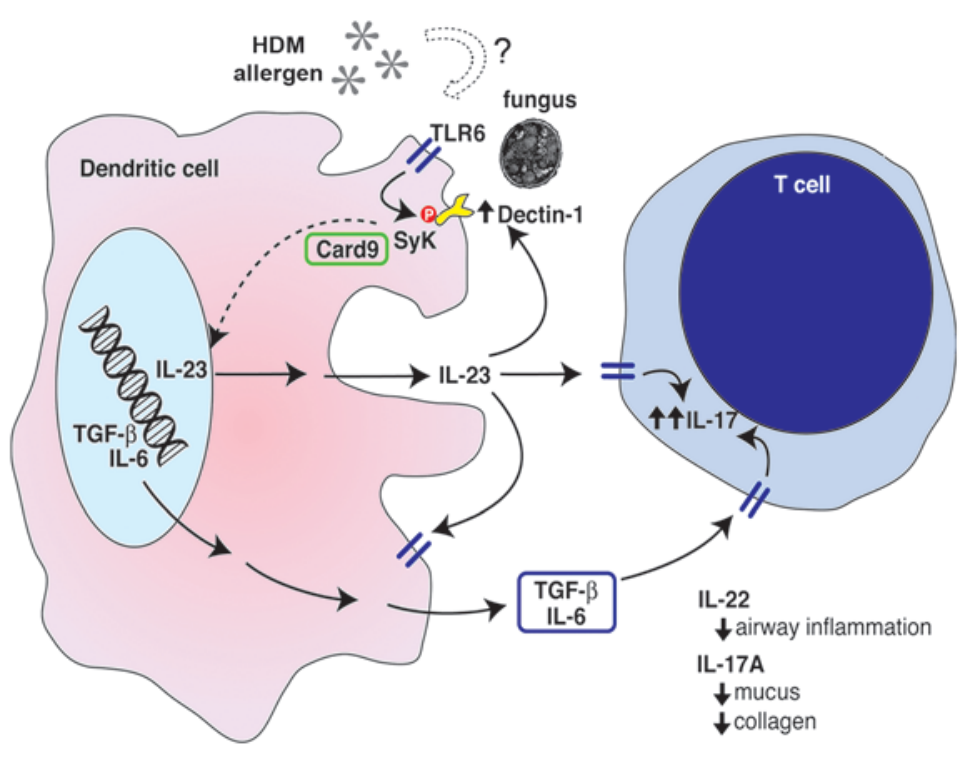

\section{Figure 9}

Schematic representation of the role of TLR6 in asthma. TLR6 activation is necessary for dectin-1 expression by DCs. TIr6 ${ }^{-/-}$DCs exhibit impaired IL-23 production due to the impairment in dectin-1 expression. Moreover, IL-23 promotes Th17 expansion by its direct effect on T cells and by increasing the release of TGF- $\beta$ and IL- 6 by DCs. TIr6 ${ }^{-/}$mice show a significant decrease in Th17 cells during chronic asthma. IL-17A participates in the control of mucus and collagen production, while IL-22 reduces inflammation. Thus, the balanced production of IL-17A and IL-22 during chronic asthma is IL-23 dependent and serves to regulate the allergic inflammatory and remodeling process. time during fungal asthma, there was no evidence that disease differences between WT and Tlr6 $6^{-1-}$ mice were a consequence of alterations in levels of this cytokine. Instead, the lack of endogenous TLR6 led to exacerbated fungal-and HDM antigen-induced asthma in mice because of the lack of sufficient IL-23 generation in the lung. Direct evidence that IL-23 was responsible for modulating the severity of fungal asthma came from our studies in which exogenous rIL-23 was delivered directly into the lungs of allergic $\operatorname{Tlr}^{-/-}$mice from day 15 to day 30 after the conidia challenge. This treatment regimen did not ameliorate airway hyperreactivity, inflammation, and remodeling in Tlr6-deficient mice, but the magnitude of these features was similar to that observed in WT asthmatic mice. These data suggest that IL-23 is not a therapeutic modality in the treatment of asthma, but rather should be viewed as an important immunoregulatory factor during experimental asthma. Indeed, excess generation of IL-23 $(26,27)$ or transgenic overexpression of IL-23 receptor (28) can exacerbate allergeninduced inflammation through increased Th2-cell and eosinophil activity and/or recruitment. In addition, excess IL-23 generation has been linked to susceptibility to fungal infection in naive mice (29). Our data differ from this previous study in that we observed that IL-23 deficiency in asthmatic $\operatorname{Tl} 6^{-1-}$ mice was associated with enhanced fungal retention, which was reversed with rIL-23 treatment in these mice. An obvious explanation is that IL-23 has unique roles in the antifungal response that are dictated by the underlying cytokine phenotype (i.e., Th1 type in naive mice and Th2 type in asthmatic mice). However, given that HDM antigen-challenged $\operatorname{Tlr}^{-/}$mice also exhibited exacerbated asthmatic responses compared with WT mice, the immune response generated by this TLR might provide a more generalized immunomodulatory effect, particularly during the chronic phases of allergic airway disease. Thus, it is clear that the role of IL-23 in the lung is complex and is dependent upon the nature, magnitude, and duration of the stimulus used to evoke lung disease.

The observation that IL-23 generation was markedly impaired in $\operatorname{Tlr}^{-1-}$ mice led us to examine how the absence of this TLR on DCs might affect its ability to generate IL-23. Not surprisingly, these cells failed to respond to a TLR2/6 ligand, but less predict- able was the finding that these DCs responded poorly to dectin-1-, TLR4-, and TLR9-specific stimuli, shedding new light on the relative importance of TLR6 for the expression and/or function of other PRRs. Consistent with these findings, we observed that other experimental or environmental allergens, including OVA, HDM antigen, and cockroach allergen, poorly activated both naive and allergen-sensitized $\operatorname{Tlr}^{-/}$DCs, as evidenced by their reduced generation of IL-23 or cell-surface expression of LAP-TGF- $\beta$ compared with that of WT DCs. Specific attention in the current study was directed toward TLR6 regulatory effects on dectin-1 expression and function on DCs, since dectin- 1 recognizes fungal $\beta$-glucans and is required for innate immune responses against germinating A. fumigatus conidia (30-33). Fungal $\beta$-glucan activation of dectin-1 leads to syk- and CARD9-dependent signaling events (34) and, ultimately, IL-23 generation in DCs $(20,35)$. Werner and colleagues (36) demonstrated the involvement of dectin- 1 in the antifungal response against $A$. fumigatus. In this study, dectin-1-deficient mice exhibited impaired production of IL-23 and IL-17, and these mice were unable to kill A. fumigatus. In our study, exogenous IL-23 contributed to a small reduction in the fungal burden in $\mathrm{Tlr}^{-/-}$allergic mice. However, at day 15 after conidia challenge, the levels of IL-17A and the number of conidia in the lungs were similar between the WT and knockout groups, suggesting that other factors in addition to the persistence of fungal conidia contribute to the increased susceptibility of $\operatorname{Tlr}^{-/-}$mice to asthma. Transcription factors including PU.1, c-Jun, and SP1 have been shown to activate the expression of surface molecules and differentiation of myeloid cells $(37,38)$. Specifically, Zhang and colleagues (38) demonstrated that PU.1 binds to the dectin-1 promoter and that the absence of PU.1 results in deficient expression of dectin-1. A finding from the present study was that exogenous rIL-23 strongly induced PU.1 and dectin-1 expression in cultured DCs. In addition, the in vivo treatment of allergic $\operatorname{Tlr}^{-/-}$mice with rIL-23 resulted in a significant increase in PU.1 in BMDCs. Thus, TLR6 has emerged as an important regulatory TLR whose expression is required for appropriate IL-23 generation by DCs and their expression of other PRRs.

Given their potential role in chronic inflammatory diseases such as RA and MS, Th17 cells have been implicated in the exacerba- 
tion of the Th2-mediated eosinophilic inflammatory response in asthma (reviewed in refs. 7, 21, 39). Experimental studies have not adequately clarified the role of IL-17A in experimental asthmatic responses, since this cytokine can negatively regulate $(40-42)$ or exacerbate $(26,43)$ features of this disease. In addition, IL-17A drives neutrophil-mediated pulmonary fibrosis in a model of bleomycin-induced fibrosis (44). Explanations for these discordant results are not immediately apparent, but the timing of synthesis (40) and the cellular source (41) of IL-17A might explain differences in these studies. In the present study, the antibody-directed targeting of IL-17A clearly exacerbated goblet cell activation and metaplasia present in the airways during the chronic phase of fungal asthma. IL-22 is generated by Th17 and other immune cells (45), and it has been shown to promote lung epithelial proliferation and resistance to injury during Klebsiella pneumoniae infection (46). IL-22 is also a negative regulator of the allergic inflammatory response (47-49), and our data confirmed that IL-22 also negatively regulates the peribronchial inflammatory response and the generation of proinflammatory IL-33 and PTX3 evoked by A. fumigatus conidia during fungal asthma (50, 51). Thus, IL-17A and IL-22 regulate different aspects of asthma, such as remodeling and inflammation (52). Both IL-17A and IL-22 transcripts were significantly increased in the lungs of $\operatorname{Tlr}^{-/-}$mice treated with rIL-23, which perhaps explains, in part, the modulating effects of rIL-23 on airway inflammation and remodeling in these mice. Another consequence of IL-23/TLR6 deficiency in this fungal asthma model was the deficient expression of LAP-TGF- $\beta$ on DCs, even though TGF- $\beta$ levels were similar in whole-lung samples from WT and $T \operatorname{lr} 6^{-/-}$mice. This cell-associated version of TGF- $\beta$ has been shown to inhibit Th1 differentiation in favor of Th17 differentiation (53). Li and colleagues also demonstrated that TGF- $\beta$ production by foxp 3 Tregs promoted Th 17 differentiation and contributed to the induction of experimental autoimmune encephalomyelitis (54). Our data showed that, besides Tregs, DCs are also important sources of TGF- $\beta$. Moreover, since WT and $\mathrm{Tlr}^{-/-}$mice exhibited similar TGF- $\beta$ production in the lungs, it is apparent that reduced TGF- $\beta$ expression by $T \operatorname{lr} 6^{-1-}$ DCs had less impact on the differentiation of Tregs, while reduced levels of IL-23, TGF- $\beta$, and IL- 6 together resulted in the deficient expansion of the Th17 population. rIL-23 treatment increased the transcripts for TGF- $\beta$ in BMDCs and the expression of TGF- $\beta$ by Tlr $^{-/-}$DCs. Thus, these data demonstrate that TLR6 is necessary for the generation of IL-23, which is required for optimal activation of DCs and induction of IL- 6 and TGF- $\beta$ expression and a corresponding expansion of Th17 cells during fungal asthma.

In summary, TLR6 has an important immunoregulatory role during experimental fungal asthma. The absence of this TLR led to clear deficits in IL-23, dectin-1, IL-17A, and IL-22 expression in asthmatic mice, which consequently exhibited exacerbated features of airway resistance, inflammation, and remodeling (Figure 9). Mechanistically, the deficit in IL-23 production and Th17 expansion appeared to be related to defects in the activation of $\operatorname{Tl} \mathrm{r}^{-/-}$DCs. Given that protective polymorphisms in clinical asthma have been identified (17) and agonists of TLR2/6 have been employed to attenuate experimental asthma (25), therapeutics targeting the activity of TLR6 might prove efficacious in the treatment of clinical asthma.

\section{Methods}

Mice. Female C57BL/6 WT mice at 6-7 weeks of age were purchased from Jackson Laboratory, and Shizuo Akira (Osaka University) provided the
$\operatorname{Tlr} 6^{-/-}$mice. All mice were maintained in a specific pathogen-free facility at the University of Michigan Medical School, and prior approval for animal experiments was obtained from the University Committee on the Use and Care of Animals.

Murine model of fungal-and HDM antigen-induced allergic asthma. Mice were sensitized via i.p. and s.c. injections of A. fumigatus antigen (Greer Laboratories) dissolved in $0.2 \mathrm{ml}$ of incomplete Freund adjuvant (IFA) (SigmaAldrich). After 3 i.n. instillations of A. fumigatus antigen, each spaced 1 week apart, mice were challenged with $5 \times 10^{6} \mathrm{~A}$. fumigatus conidia via an i.t. injection. $\operatorname{Tl} 6^{--}$mice were treated every other day with $620 \mathrm{ng}$ of rIL-23 (eBioscience) or $25 \mu \mathrm{g}$ of anti-mouse IL-17A antibody, anti-mouse IL-22 antibody, or the appropriate IgG control i.n. from days 15 to 21 or 15 to 30 after conidia challenge. To generate HDM antigen-induced allergic asthma, mice were challenged for 5 days a week with $10 \mu$ l of HDM antigen (Greer Laboratories) administered i.n. for a total of 6 weeks. At days 15 and 30 after conidia challenge or 48 and 72 hours after the last i.n. challenge with HDM antigens, bronchial resistance was assessed in a Buxco plethysmograph (Buxco) after i.v. methacholine injection $(420 \mu \mathrm{g} / \mathrm{kg})$.

Isolation and culture of lung DCs and BMDCs. To obtain lung DCs, total lung cells were collected from WT (naive) mice by enzymatic digestion, and the populations of DCs were differentiated from alveolar macrophages (AMO) and interstitial macrophages (IMO) by the expression of $\mathrm{CD} 11 \mathrm{c}$ and $\mathrm{F} 4 / 80$ (i.e., $\mathrm{CD} 11 \mathrm{c}^{+} \mathrm{F} 4 / 80^{-}$for $\mathrm{DCs}, \mathrm{CD} 11 \mathrm{c}^{+} \mathrm{F} 4 / 80^{+}$for $\mathrm{AMO}$, and $\mathrm{CD} 11 \mathrm{c}^{-} \mathrm{F} 4 / 80^{+}$for IMO). To obtain BMDCs, BM cells were isolated from WT and $\mathrm{Tlr}^{-/-}$mice by flushing femurs with $5 \mathrm{ml}$ PBS. The cells were cultured for 6 days in RPMI 1640 supplemented with $100 \mathrm{U} / \mathrm{ml}$ penicillin, $100 \mu \mathrm{g} / \mathrm{ml}$ streptomycin, $5 \mathrm{mM}$ glutamine, and 10\% FBS and supplemented with $10 \mathrm{ng} / \mathrm{ml}$ of GM-CSF at $37^{\circ} \mathrm{C}$ with $5 \% \mathrm{CO}_{2}$. Magnetic bead-purified (Miltenyi Biotec) $\mathrm{CD} 11 \mathrm{c}^{+}$cells were stimulated with one of the following: $2.5 \mu \mathrm{g} / \mathrm{ml}$ PAM3cys (EMC Microcollections), $2.5 \mu \mathrm{g} / \mathrm{ml}$ of PAM2cys, $10 \mu \mathrm{g} / \mathrm{ml}$ of Poly-IC, $1 \mu \mathrm{g} / \mathrm{ml}$ LPS (E. coli 0111:B4; SigmaAldrich), 2 nM mouse CpG-DNA (Hycult Biotechnology), $50 \mu \mathrm{g} / \mathrm{ml}$ zymosan, $1 \mu \mathrm{g} / \mathrm{ml}$ curdlan (Sigma-Aldrich), or $10 \mathrm{ng} / \mathrm{ml} \mathrm{rIL-23} \mathrm{or} \mathrm{rIL-12} \mathrm{(eBio-}$ science). Other cells were cultured with OVA $(10 \mu \mathrm{g} / \mathrm{ml})$, HDM antigen (0.02 units $/ \mathrm{ml})$, and cockroach antigens $(10 \mu \mathrm{g} / \mathrm{ml})$. After 6 or 24 hours, total RNA was isolated using TRIzol reagent (Invitrogen). After 24 hours of stimulation, cell-free supernatant from each sample was collected and ELISA was used to measure cytokine levels.

Real-time TaqMan analysis. Total RNA was obtained from cells or lung homogenate using TRIzol (Invitrogen) according to the manufacturer's directions. RNA was reverse-transcribed into cDNA using a $25 \mu \mathrm{l}$ reaction mixture containing the $1 \times$ First-Strand cDNA Synthesis Kit (Invitrogen), including $250 \mathrm{ng}$ oligo(dT) ${ }_{12-18}$ primer, $1.6 \mathrm{mM}$ dNTPs, 5 U RNase inhibitor, and 100 U Moloney murine virus RT. A TaqMan 7500 sequence detection system (Applied Biosystems) was used for real-time quantitative TaqMan PCR analysis. GAPDH (Applied Biosystems) was used as internal control.

Lung histological and immunohistochemistry analysis. Lung biopsies from asthmatic and nonasthmatic patients $(n=6$ asthmatic and $n=6$ nonasthmatic patients) were deidentified and analyzed using routine immunohistochemical techniques. Approval to use these lung tissue samples was obtained from an institutional review board at the University of British Columbia, and patients gave informed consent. Whole lungs were fully inflated with $10 \%$ formalin, dissected, and placed in fresh $10 \%$ formalin for 24 hours. Routine histological techniques were used to paraffin embed the entire lung, and 5- $\mu \mathrm{m}$ sections of whole lung were stained with $\mathrm{H} \& \mathrm{E}$ or Masson trichrome. TLR6 and dectin- 1 expression were analyzed by immunohistochemistry or immunofluorescence using biotinylated antimouse dectin-1 (Cell Sciences) or anti-TLR6 (Novus Biologicals) antibody. An HRP-aminoethylcarbazole cell and tissue staining kit was used according to the manufacturer's instructions (R\&D Systems). Quantitative digi- 
tal morphometric analysis of the number of cells and percentage of collagen was performed using the application program IP Lab Spectrum-R4a. A minimum of 15 images per slide were analyzed in each whole-lung tissue section. Percentage of collagen was then calculated by dividing the total pixel area by the pixel area corresponding to collagen.

Analysis of cytokine levels. The levels of cytokines were measured in lung homogenates or supernatant of DC culture from asthmatic mice either by standard sandwich ELISA or via a bead-based multiple target sandwich ELISA system (BIOPLEX; Bio-Rad Laboratories). Before ELISA, snap-frozen lung samples were thawed on ice and homogenized in a solution containing $2 \mathrm{mg}$ of complete protease inhibitor (Boehringer Mannheim) per $\mathrm{ml}$ of normal saline. All cytokines were normalized to the total protein concentration in the tissue, as determined by the Bradford method (Bio-Rad Laboratories).

Flow cytometric analysis. Spleen or LN samples were obtained from allergic mice at day 21 or day 30 after conidia challenge and incubated with collagenase (Roche) at $37^{\circ} \mathrm{C}$ for 40 minutes. Total cells from spleen or magnetic beads (Miltenyi Biotec) were used to sort $\mathrm{CD} 11 \mathrm{c}^{+}$and $\mathrm{CD} 4^{+}$cells, and these cells were cocultured for 72 hours with medium, A. fumigatus antigen, or antigen plus anti-dectin-1 (Hycult Biotechnology). Cell suspensions were then incubated with anti-CD16/32 (2.4G2) and further labeled with fluorescent dye-conjugated $\mathrm{mAb}(\mathrm{CD} 4$; CD3; CD11b, CD11c; BD Biosciences or eBioscience). Following the staining of surface markers, cells were fixed, permeabilized (BD Cytofix/Cytoperm Kit; BD Biosciences - Pharmingen), and stained for intracellular IFN- $\gamma$, IL-4, IL-17, foxp3, and TGF- $\beta$ (BD Bio- sciences - Pharmingen or eBioscience). Samples were acquired on an LSRII machine using FACSDiVa software (BD Biosciences). Data were analyzed using FlowJo software (Tree Star Inc.).

Statistics. Statistical significance was determined using the unpaired Student's $t$ test or ANOVA followed by Tukey's test for multiple comparisons. $P \leq 0.05$ was considered statistically significant. Calculations were performed using the Prism 4.0 software program (GraphPad Software).

\section{Acknowledgments}

The authors thank Ana Lucia Coelho and Lisa Riggs for their technical support, Robin Kunkel for assistance with graphic illustration, Judith Connett for critically editing the manuscript, and Carlos Henrique Serezani and Matthew A. Schaller for helpful discussion.

Received for publication September 3, 2010, and accepted in revised form September 14, 2011.

Address correspondence to: Cory Hogaboam, Department of Pathology, University of Michigan Medical School, Room 4057, BSRB, 109 Zina Pitcher Place, Ann Arbor, Michigan 48109-2200, USA. Phone: 734.647.8153; Fax: 734.615.8166; E-mail: hogaboam@ med.umich.edu. Or to: Ana Paula Moreira, Department of Pathology, University of Michigan Medical School, Room 4658, BSRB, 109 Zina Pitcher Place, Ann Arbor, Michigan 48109-2200, USA. Phone: 734.936.1856; Fax: 734.936.7996; E-mail: anapaula@umich.edu.
1. Galli SJ, Tsai M, Piliponsky AM. The development of allergic inflammation. Nature. 2008; 454(7203):445-454.

2. Denning DW, O'Driscoll BR, Hogaboam CM, Bowyer P, Niven RM. The link between fungi and severe asthma: a summary of the evidence. Eur Respir J. 2006;27(3):615-626.

3. Agarwal R, Gupta D. Severe asthma and fungi: current evidence. Med Mycol. 2011;49(suppl 1):S150-S157.

4. Lambrecht BN. Lung dendritic cells: targets for therapy in allergic disease. Curr Mol Med. 2008; 8(5):393-400.

5. Hammad H, Lambrecht BN. Recent progress in the biology of airway dendritic cells and implications for understanding the regulation of asthmatic inflammation. J Allergy Clin Immunol. 2006; 118(2):331-336.

6. Barnes PJ. Immunology of asthma and chronic obstructive pulmonary disease. Nat Rev Immunol. 2008;8(3):183-192.

7. Finkelman FD, Hogan SP, Hershey GK, Rothenberg ME, Wills-Karp M. Importance of cytokines in murine allergic airway disease and human asthma. Jimmunol. 2010;184(4):1663-1674.

8. O'Driscoll BR, Hopkinson LC, Denning DW. Mold sensitization is common amongst patients with severe asthma requiring multiple hospital admissions. BMC Pulm Med. 2005;5:4.

9. Denning DW, et al. Randomized controlled trial of oral antifungal treatment for severe asthma with fungal sensitization: The Fungal Asthma Sensitization Trial (FAST) study. Am J Respir Crit Care Med. 2009; 179(1):11-18.

10. Phipps S, Lam CE, Foster PS, Matthaei KI. The contribution of toll-like receptors to the pathogenesis of asthma. Immunol Cell Biol. 2007;85(6):463-470.

11. Kurup VP, Raju R, Manickam P. Profile of gene expression in a murine model of allergic bronchopulmonary aspergillosis. Infect Immun. 2005;73(7):4381-4384.

12. Ozinsky A, et al. The repertoire for pattern recognition of pathogens by the innate immune system is defined by cooperation between toll-like receptors. Proc Natl Acad Sci U S A. 2000;97(25):13766-13771.

13. Horng T, Barton GM, Flavell RA, Medzhitov R.
The adaptor molecule TIRAP provides signalling specificity for Toll-like receptors. Nature. 2002; 420(6913):329-333.

14. Tantisira K, et al. Toll-like receptor 6 gene (TLR6): single-nucleotide polymorphism frequencies and preliminary association with the diagnosis of asthma. Genes Immun. 2004;5(5):343-346.

15. Hoffjan S, et al. Evaluation of the toll-like receptor 6 Ser249Pro polymorphism in patients with asthma, atopic dermatitis and chronic obstructive pulmonary disease. BMC Med Genet. 2005;6:34.

16. Puthothu B, Heinzmann A. Is toll-like receptor 6 or toll-like receptor 10 involved in asthma genetics--or both? Allergy. 2006;61(5):649-650.

17. Kormann MS, et al. Toll-like receptor heterodimer variants protect from childhood asthma. J Allergy Clin Immunol. 2008;122(1):86-92.

18. Kesh S, et al. TLR1 and TLR6 polymorphisms are associated with susceptibility to invasive aspergillosis after allogeneic stem cell transplantation. Ann N Y Acad Sci. 2005;1062:95-103.

19. Chun E, et al. Toll-like receptor expression on peripheral blood mononuclear cells in asthmatics; implications for asthma management. J Clin Immunol. 2010;30(3):459-464.

20. LeibundGut-Landmann S, et al. Syk- and CARD9dependent coupling of innate immunity to the induction of $\mathrm{T}$ helper cells that produce interleukin 17. Nat Immunol. 2007;8(6):630-638.

21. Mucida D, Salek-Ardakani S. Regulation of TH17 cells in the mucosal surfaces. J Allergy Clin Immunol. 2009;123(5):997-1003.

22. Takeuchi O, et al. TLR6: A novel member of an expanding toll-like receptor family. Gene. 1999; 231(1-2):59-65.

23. Fuchs B, et al. A Toll-like receptor $2 / 6$ agonist reduces allergic airway inflammation in chronic respiratory sensitisation to Timothy grass pollen antigens. Int Arch Allergy Immunol. 2010;152(2):131-139.

24. Patel M, et al. TLR2 agonist ameliorates established allergic airway inflammation by promoting Th1 response and not via regulatory T cells. J Immunol. 2005;174(12):7558-7563.

25. Weigt H, Nassenstein C, Tschernig T, Muhlradt
PF, Krug N, Braun A. Efficacy of macrophage-activating lipopeptide- 2 combined with interferongamma in a murine asthma model. Am J Respir Crit Care Med. 2005;172(5):566-572.

26. Wakashin H, et al. IL-23 and Th17 cells enhance Th2-cell-mediated eosinophilic airway inflammation in mice. Am J Respir Crit Care Med. 2008; 178(10):1023-1032.

27. Lewkowich IP, Lajoie S, Clark JR, Herman NS, Sproles AA, Wills-Karp M. Allergen uptake, activation, and IL-23 production by pulmonary myeloid DCs drives airway hyperresponsiveness in asthmasusceptible mice. PLoS One. 2008;3(12):e3879.

28. Peng J, Yang XO, Chang SH, Yang J, Dong C. IL23 signaling enhances Th2 polarization and regulates allergic airway inflammation. Cell Res. 2010; 20(1):62-71.

29. Zelante T, et al. IL-23 and the Th17 pathway promote inflammation and impair antifungal immune resistance. Eur J Immunol. 2007;37(10):2695-2706.

30. Steele C, et al. The beta-glucan receptor dectin-1 recognizes specific morphologies of Aspergillus fumigatus. PLoS Pathog. 2005;1(4):e42.

31. Hohl TM, et al. Aspergillus fumigatus triggers inflammatory responses by stage-specific beta-glucan display. PLoS Pathog. 2005;1(3):e30.

32. Gersuk GM, Underhill DM, Zhu L, Marr KA. Dectin-1 and TLRs permit macrophages to distinguish between different Aspergillus fumigatus cellular states. J Immunol. 2006;176(6):3717-3724.

33. Taylor PR, et al. Dectin-1 is required for beta-glucan recognition and control of fungal infection. Nat Immunol. 2007;8(1):31-38.

34. Hancock MK, Kopp L, Bi K. High-throughput screening compatible cell-based assay for interrogating activated notch signaling. Assay Drug Dev Technol. 2009;7(1):68-79.

35. Selman AE, Copeland LJ. Secondary cytoreductive surgery in the patient with recurrent ovarian cancer is often beneficial. Crit Rev Oncol Hematol. 2002; 41(3):335-341.

36. Werner JL, et al. Requisite role for the dectin-1 betaglucan receptor in pulmonary defense against Aspergillus fumigatus. J Immunol. 2009;182(8):4938-4946. 
37. Eichbaum Q, et al. Murine macrophage mannose receptor promoter is regulated by the transcription factors PU.1 and SP1. Blood. 1997;90(10):4135-4143.

38. Zhang C, et al. Downregulation of PU.1 leads to decreased expression of Dectin-1 in alveolar macrophages during Pneumocystis pneumonia. Infect Immun. 2010;78(3):1058-1065.

39. Nakajima H, Hirose K. Role of IL-23 and Th17 Cells in Airway Inflammation in Asthma. Immune Netw. 2010;10(1):1-4.

40. Schnyder-Candrian S, et al. Interleukin-17 is a negative regulator of established allergic asthma. J Exp Med. 2006;203(12):2715-2725.

41. Murdoch JR, Lloyd CM. Resolution of allergic airway inflammation and airway hyperreactivity is mediated by IL-17-producing \{gamma\} $\{$ delta\}T cells. Am J Respir Crit Care Med. 2010;182(4):464-476.

42. Moreira AP, et al. Serum amyloid P attenuates M2 macrophage activation and protects against fungal spore-induced allergic airway disease. J Allergy Clin Immunol. 2010;126(4):712-721.
43. Lajoie S, et al. Complement-mediated regulation of the IL-17A axis is a central genetic determinant of the severity of experimental allergic asthma. Nat Immunol. 2010;11(10):928-935.

44. Wilson MS, et al. Bleomycin and IL-1beta-mediated pulmonary fibrosis is IL-17A dependent. J Exp Med. 2010;207(3):535-552.

45. Zenewicz LA, Flavell RA. IL-22 and inflammation: leukin' through a glass onion. Eur J Immunol. 2008;38(12):3265-3268.

46. Aujla SJ, et al. IL-22 mediates mucosal host defense against Gram-negative bacterial pneumonia. Nat Med. 2008;14(3):275-281.

47. Schnyder B, Lima C, Schnyder-Candrian S. Interleukin-22 is a negative regulator of the allergic response. Cytokine. 2010;50(2):220-227.

48. Souwer Y, Szegedi K, Kapsenberg ML, de Jong EC. IL-17 and IL-22 in atopic allergic disease. Curr Opin Immunol. 2010;22(6):821-826.

49. Besnard AG, et al. Dual Role of IL-22 in allergic airway inflammation and its cross-talk with IL-17A.
Am J Respir Crit Care Med. 2011;183(9):1153-1163.

50. Hollan I, et al. Increased levels of serum pentraxin 3 , a novel cardiovascular biomarker, in patients with inflammatory rheumatic disease. Arthritis Care Res (Hoboken). 2010;62(3):378-385.

51. Mauri T, et al. Persisting high levels of plasma pentraxin 3 over the first days after severe sepsis and septic shock onset are associated with mortality. Intensive Care Med. 2010;36(4):621-629.

52. Eyerich S, Eyerich K, Cavani A, Schmidt-Weber C. IL-17 and IL-22: siblings, not twins. Trends Immunol. 2010;31(9):354-361.

53. Gandhi R, Anderson DE, Weiner HL. Cutting Edge: Immature human dendritic cells express latencyassociated peptide and inhibit $\mathrm{T}$ cell activation in a TGF-beta-dependent manner. J Immunol. 2007; 178(7):4017-4021.

54. Li MO, Wan YY, Flavell RA. T cell-produced transforming growth factor-beta1 controls $\mathrm{T}$ cell tolerance and regulates Th1- and Th17-cell differentiation. Immunity. 2007;26(5):579-591. 